\title{
Modelling non-isothermal absorption of vapour into expanding liquid sheets
}

\author{
A. Acosta-Iborra *, N. García, D. Santana \\ Departamento de Ingeniería Térmica y de Fluidos, Universidad Carlos III de Madrid, Avda. Universidad 30, 28911 Leganés, Madrid, Spain
}

\begin{abstract}
A B S T R A C T
The problem of non isothermal absorption of vapour into freely expanding liquid sheets is addressed in this study. This is done in the context of four models that characterise the coupled heat and mass transfer in the liquid phase: a nonlinear model retaining the effect of sheet growth, an approximate model for slowly increasing mass flow rate in the sheet, a large Lewis number model and finally, a boundary layer model. These models have been numerically or analytically solved and applied to the comparative anal ysis of two different working pairs, $\mathrm{LiBr} \mathrm{H}_{2} \mathrm{O}$ and $\mathrm{LiNO}_{3} \mathrm{NH}_{3}$, under conditions representative of adia batic absorption in refrigeration systems. The limits of applicability of each model have been assessed and the sensitivity of the results to the sheet aperture angle, heat of absorption and initial subcooling has also been tested. For equal initial mass fraction and subcooling, the models indicate that Sherwood number and the rate of absorption in laminar expanding sheets for the $\mathrm{LiNO}_{3} \mathrm{NH}_{3}$ solution are always superior to those for the $\mathrm{LiBr} \mathrm{H}_{2} \mathrm{O}$ solution.
\end{abstract}

\section{Introduction}

Absorption chillers and heat pumps have received growing attention over the last few decades. These machines allow the use of low grade heat to produce a cooling effect, or a heat trans formation to higher temperature levels, with no (or minimal) mechanical power consumption [1,2]. This makes them an attrac tive option in waste heat recovery, solar cooling and energy saving policies.

The absorber is one of the most performance limiting and vol ume demanding components of an absorption system. In the ab sorber the concentrated liquid solution absorbs the refrigerant vapour that comes from the evaporator. During this process, heat and mass transfer in the liquid solution are strongly coupled. Sev eral configurations for the liquid and vapour streams in the absor ber have been proposed: falling films, bubbles, sprays and droplets, liquid jets and sheets, etc. Absorption using drops, sprays, liquid jets and sheets takes place in an adiabatic chamber far from iso thermal conditions. The cooling of the solution is carried out sepa rately, in a specialised and efficient heat exchanger. This operation mode is generally referred to as adiabatic absorption [3].

The use of expanding liquid sheets in adiabatic absorbers may offer advantages in enhancing the mass transfer as well as in reducing both volume and cost of the system. In this work, follow ing the terminology of Simpson and Lynn [4], an expanding liquid sheet refers to any free stream of solution that expands in multiple directions from one common origin, while its thickness progres

\footnotetext{
* Corresponding author. Fax: +34 916249430.

E-mail address: aacosta@ing.uc3m.es (A. Acosta-Iborra).
}

sively reduces. Flat fan sheets, conical sheets and radially expand ing sheets are significant examples of this flow configuration. A smaller injection pressure is required to produce an expanding sheet than is required to atomize the liquid in a spray. There is a broad literature concerning the dynamics of expanding liquid sheets. Classical studies of these spreading sheets can be found in [5 9].

Several works have been conducted in order to understand the heat and mass transfer in expanding liquid sheets; however, most of these studies are not directly devoted to absorption chillers and heat pumps.

One of the earliest experimental works attending the problem of water vapour condensation on subcooled conical swirling sheets was that performed by Weinberg in 1952 [10]. In this work, large temperature variations, and therefore high rates of condensation, were observed in the sheet region of the spray. Other subsequent investigations on condensation also addressed the direct measure ment of temperatures in conical sheets of water [11,12] and R113 refrigerant [13]. Condensation on laminar flat fan sheets was stud ied by Hasson et al. [14] and Tamir and Hasson [15]. This second work also examines the evaporation process in flat fan sheets.

The seminal work of Hasson et al. in 1964 [16] includes the first analytical approximation to the problem of heat transfer in a smooth fan shaped sheet of water during vapour condensation. The energy equation for flat fan sheets was resolved in the form of a series solution, neglecting the effect of mass growth due to condensation at the sheet surface. Comparison of these results to previous measurements $[14,15]$ demonstrates a qualitative simi larity. Lee and Takin [11] developed a numerical simulation of the condensation in the laminar sheet region of a conical spray 


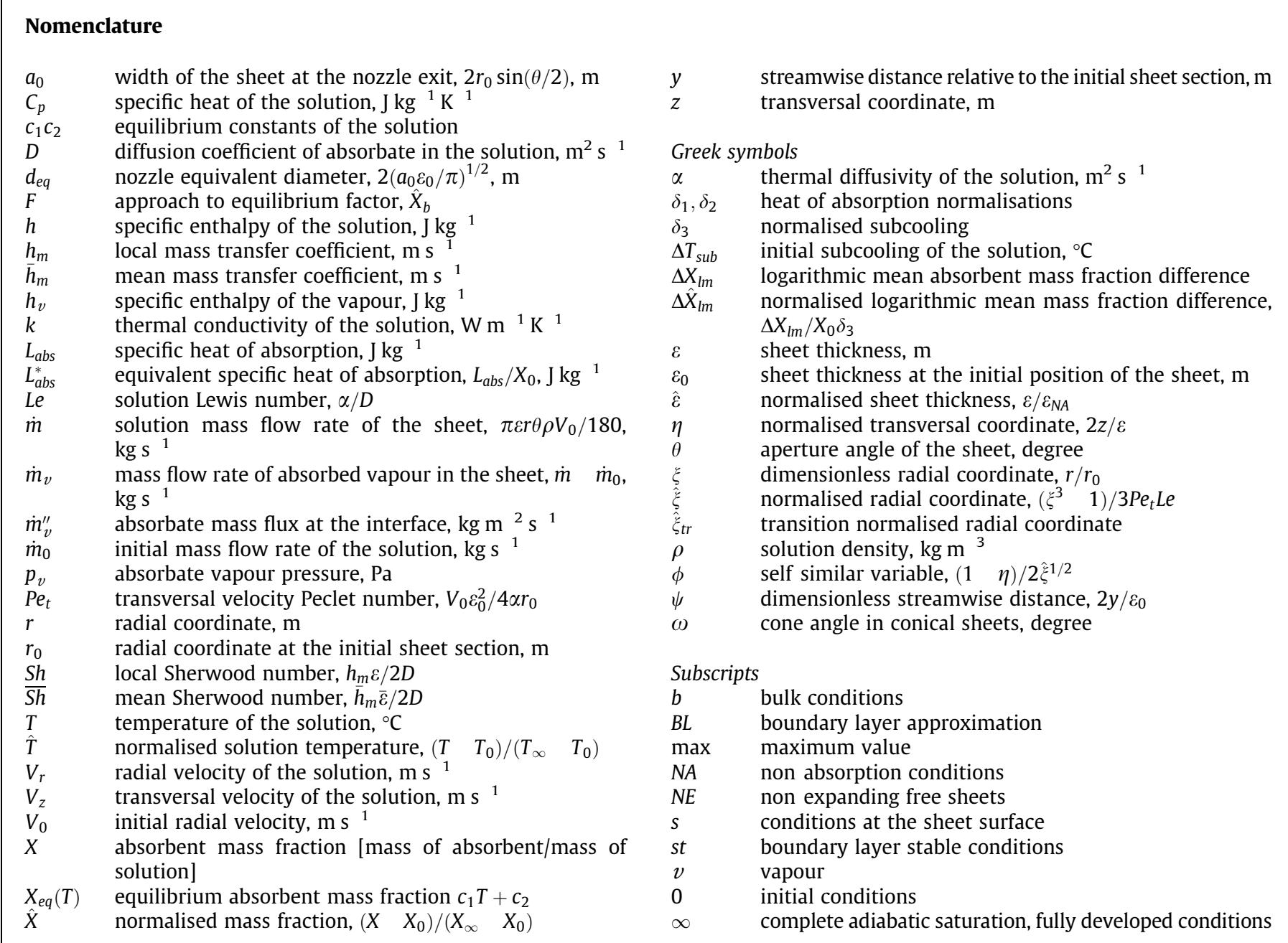

whose aperture angle and velocity had been previously obtained in [17]. More recently, Takahashi and co workers [12] extended the series solution of Hasson et al. [16] to the case of condensation in a swirling conical sheet. In all of these works, the predicted mean temperature in the sheet underestimated the experimental data. Turbulence transport in the sheet was suggested as a factor that could produce such discrepancies [12].

In 1972 Tamir and Rachmilev [18] published their experimental results on absorption of $\mathrm{CO}_{2}$ in a "rapid laminar fan shaped water sheet". The temperature in the sheet remained constant because of the low heat of absorption of $\mathrm{CO}_{2}$ into water. Using the analogy be tween heat and mass transfer, Tamir and Rachmilev [18] adapted the series solution for the flat fan sheets of Hasson et al. [16] to the problem of isothermal absorption. Their analytical model was found to be in good agreement with measurements. Simpson and Lynn [4] experimentally characterised the desorption of various substances such as oxygen, Freon 114 and $n$ butane, from laminar flat fan and conical sheets of water/salt solutions. A similar conver sion of the Hasson et al. [16] series solution was also performed by Simpson and Lynn [4] in their model estimation of isothermal desorption, and reasonable concordance between the model and the experimental data was found. For turbulent expanding sheets however, the concordance was only qualitative, necessitating a corrected empirical model [19]. In all of these works on absorption and desorption, conditions near equilibrium were detected even at extremely short sheet lengths.

In a work dealing with the problem of water vapour absorption into sprays of aqueous lithium bromide solution, Ryan et al. [20] indicated that the short sheet region of a spray before breaking into droplets had a relevant contribution in their measurements of the global absorption in conical and flat fan sprays. Neglecting the sheet thickness variation they used a sheet of constant thickness (from [16]) in order to incorporate the contribution of the short sheet region into the spray absorption modelling.

The aim of the present work is to extend the aforementioned numerical and analytical models for expanding sheets to the case of non isothermal vapour absorption. Conditions representative of adiabatic absorbers in refrigeration systems are selected to the integration of the equations. The coupled heat and mass transfer models presented in this investigation are also inspired by the sim ulation techniques developed for absorption in falling films. Excel lent reviews, containing numerical, analytical and experimental data for absorption in falling films can be found in the works of Grossmann [21] and Killion and Garimella [22].

\section{Expanding sheet adiabatic absorption}

Fig. 1 presents a conceptual drawing of an expanding sheet adi abatic absorber. It consists of an adiabatic chamber into which the concentrated solution of an absorption system is injected, produc ing an expanding liquid stream such as a flat fan, conical, or radi ally expanding sheet (see Fig. 1a, b and c, respectively). In Fig. 1c, a $360^{\circ}$ nozzle generates the disk sheet, but other methods can also be used (for example the impingement of two round liquid jets). The liquid solution is composed of two substances: absorbent and absorbate (also known as refrigerant). Additionally, there is a 


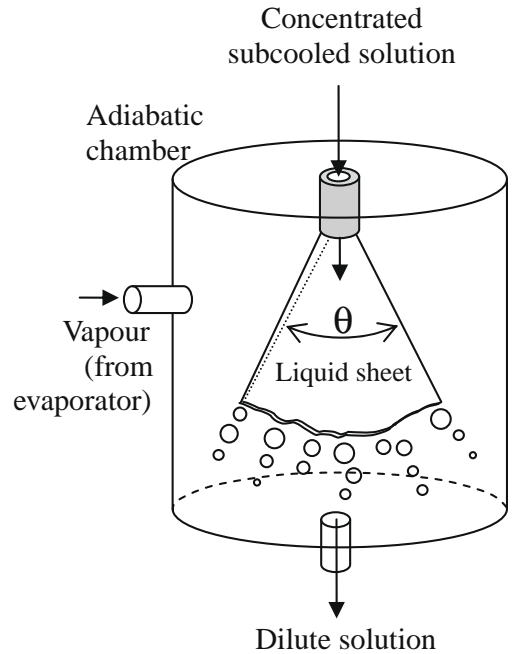

(a)

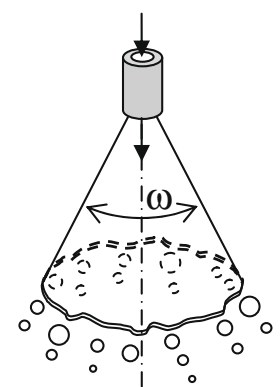

(b)

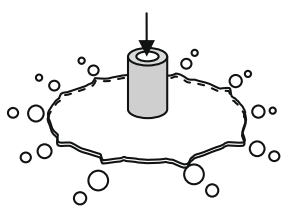

(c)
Fig. 1. Schematic diagram of an adiabatic absorber containing a flat-fan sheet of liquid solution (a). Other liquid flow configurations are: conical sheet (b), and disk sheet (c).

flow of absorbate that is in the vapour phase, close to saturation conditions, that enters the chamber. Absorbate vapour comes from the evaporator of the absorption machine where it acts as a refrig erant working fluid.

The liquid solution enters the chamber in a subcooled state; that is, its temperature is initially less than the equilibrium tem perature at the pressure $p_{v}$ of absorbate vapour in the chamber. As a result, the absorbate vapour is spontaneously absorbed by the liquid sheet, decreasing the solution absorbent mass fraction from the input (concentrated solution) to the output (diluted solu tion) of the absorber.

In this work, results will be compared between two different working pairs of binary solution: aqueous lithium bromide solution, $\mathrm{LiBr} \mathrm{H}_{2} \mathrm{O}$; and lithium nitrate ammonia solution, $\mathrm{LiNO}_{3} \mathrm{NH}_{3}$. Here $\mathrm{H}_{2} \mathrm{O}$ and $\mathrm{NH}_{3}$ are the absorbate (or refrigerant) substances and the remaining substances the absorbent. Table 1 contains basic properties of these working pairs. Properties for the pair $\mathrm{LiNO}_{3} \mathrm{NH}_{3}$ correspond to the operating conditions of a low pressure double stage absorption chiller fed with a low grade heat source [23]. Working conditions for the pair $\mathrm{LiBr} \mathrm{H}_{2} \mathrm{O}$ [24 26], have been chosen so that the solution operates with the same inlet temperature $T_{0}$ and mass fraction $X_{0}$ as the ammonia solution pair. Mass diffusivity $D$ of water into $\mathrm{LiBr} \mathrm{H}_{2} \mathrm{O}$ solution is taken from [27].

Table 1

Working pairs properties corresponding to similar initial conditions $\left(T_{0}=40^{\circ} \mathrm{C}\right.$ and $\left.X_{0} \quad 0.633\right)$.

\begin{tabular}{lll}
\hline & $\mathrm{LiBr}-\mathrm{H}_{2} \mathrm{O}$ & $\mathrm{LiNO}_{3}-\mathrm{NH}_{3}$ \\
\hline$p_{v}[\mathrm{~Pa}]$ & $9.3 \times 10^{2}$ & $2.36 \times 10^{5}$ \\
$\rho\left[\mathrm{kg} \mathrm{m}^{3}\right]$ & 1789 & 1132 \\
$C_{p}\left[\mathrm{~J} \mathrm{~kg}^{1}{ }^{1}{ }^{1}\right]$ & 1830 & 2620 \\
$\alpha\left[\mathrm{m}^{2} \mathrm{~s}^{1}\right]$ & $1.37 \times 10^{7}$ & $5.07 \times 10^{7}$ \\
$D\left[\mathrm{~m}^{2} \mathrm{~s}^{1}\right]$ & $1.54 \times 10^{9}$ & $0.74 \times 10^{9}$ \\
$c_{1}\left[{ }^{\circ} \mathrm{C}^{1}\right]$ & $4.85 \times 10^{3}$ & $3.72 \times 10^{3}$ \\
$c_{2}[-]$ & 0.385 & 0.434 \\
$L_{a b s}\left[\mathrm{~J} \mathrm{~kg}^{1}\right]$ & $2.44 \times 10^{6}$ & $1.31 \times 10^{6}$ \\
\hline
\end{tabular}

\section{Physical model and general assumptions}

The physical model to be considered in the simulation is sche matically represented in Fig. 2. A free expanding sheet of liquid solution flows from an injector, a nozzle or a small impinging plate. Both sides of the liquid sheets are in contact with a stagnant absor bate (refrigerant) in the vapour phase. This is the only gaseous sub stance in the chamber. The liquid sheet spreads outwardly in divergence directions from a virtual origin. Expansion of the flow causes the thickness $\varepsilon$ of the liquid sheet to gradually narrow in the stream direction. The sheet exits the nozzle or injector at $r \quad r_{0}$, from this precise moment it starts to absorb the surrounding vapour.

When the divergence directions of the flow are in a plane, as they are in Fig. 2, the liquid sheet adopts the shape of a flat fan or a radially expanding disk (the latter if the angle $\theta$ covered by the sheet reaches $360^{\circ}$ ). Conical sheets are created if the directions of divergence form a hollow cone. This can be approximated by a flat fan sheet rolled up along the axis of a cone whose angle $\omega$ (see Fig. 1b) satisfies $\omega 2 \arcsin \left(\theta / \theta_{\max }\right)$, being $\theta_{\max }=360^{\circ}$.

The mass absorption into the expanding sheet of Fig. 2, through two sides of the sheet, is strongly coupled with the transfer of heat of absorption that is released at the surface. In formulating the absorption model the following assumptions are made:

- Steady state conditions are assumed.

- The liquid sheet flow is laminar and its velocity is high enough to neglect the effects of the gravitational acceleration as well as interfacial liquid vapour drag on the sheet hydrodynamics.

- The contribution of the borders of the sheet (rims) to the total amount of absorbed refrigerant is neglected.

- Finite dilution at the interface, transversal velocity $V_{z}$, and growth of mass rate in the sheet due to absorption are considered.

- At the free surface of the sheet, instantaneous equilibrium arises between the solution temperature and the species mass fraction.

- At the initial position of the sheet, $r \quad r_{0}$, the temperature and mass fraction profiles of the solution are uniform and equal to $T_{0}$ and $X_{0}$, respectively. This holds except at the sheet surface where the mass fraction decreases to $X_{e q}\left(T_{0}\right)$ on account of the equilibrium condition.

- The mixture properties $\left(\rho, C_{p}, \alpha, D\right)$ and heat of absorption are considered to be constant under the narrow range of tempera tures and species mass fractions reached by the present model.

- Heat transfer from the solution to the vapour (or to its surround ings) and viscous thermal dissipation are neglected.

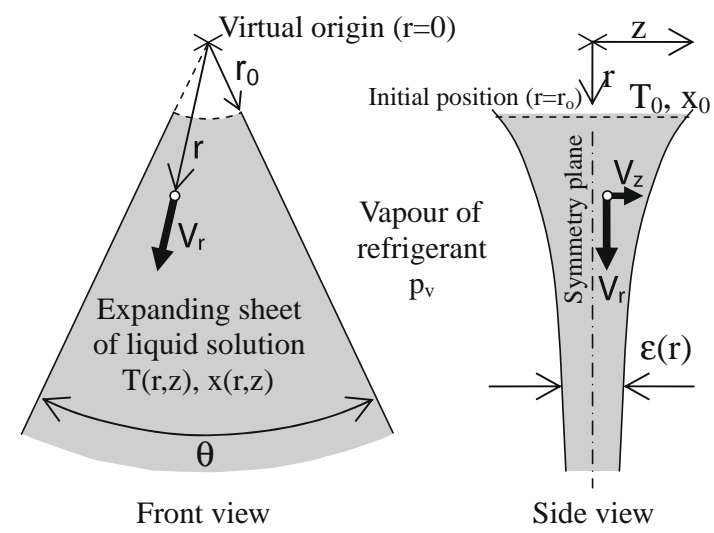

Fig. 2. Physical model and coordinate system. 
Many of these simplifying assumptions are representative of prac tical operating conditions in adiabatic absorption and are also com mon to many models in falling film absorption. At this regard, it is important to note that in non adiabatic absorption large incre ments of absorbate mass fraction and temperature can occur, being in that case recommendable to calculate the local variation of working pair properties with $X$ and $T$ (see for example [28]). This contrasts with adiabatic absorption for which the assumption of unvarying properties in the mixture is particularly suited because potential increments of $T$ and, above all, $X$ are significantly smaller $[3,23]$, as demonstrated in Appendix A.

\section{Flow field in the sheet}

Because it is assumed constant density in the flow, the liquid sheet hydrodynamics do not depend on temperature and species mass fraction. Therefore, the velocity field in an expanding sheet can be deduced at the onset for later use in the heat and mass transfer equations. Owing to stream wise momentum conserva tion and assuming invariance in angular direction, the radial veloc ity $V_{r}$ of the mixture in a smooth laminar expanding sheet can be considered constant $[4,6,1518,29]$ :

$V_{r} \quad V_{0}$

where $V_{0}$ is the radial initial velocity of the liquid sheet at $r \quad r_{0}$. Thus, from the continuity equation in polar coordinates and consid ering null angular velocity, it is straightforward to obtain the trans versal velocity $V_{z}$ of the mixture in the sheet:

$V_{z} \quad \frac{V_{0} Z}{r}$

where $z$ is the transversal coordinate (Fig. 2). Observe that $V_{z}$ is null at the sheet symmetry plane $z \quad 0$ and negative for $z>0$. This means that, given any point in the sheet, an incoming flow of solu tion in the transversal direction arises in order to replace the solu tion that is expanding radially in the sheet.

It should be noted that sheet retraction at the rims [30] and dis integration [5] has not been considered. As a result, the model should only be applied to smooth flow conditions in the region be fore sheet instabilisation and break up.

\section{Energy and mass fraction equations}

\subsection{Complete nonlinear model}

Transport of heat and mass within the liquid sheet is gov erned by advective and diffusive processes. No generation of spe cies takes place in the problem and diffusion of heat and mass in the sheet in the streamwise direction can be disregarded as com pared to diffusion in the transverse direction. Under these sim plifications and the assumptions presented previously, the differential equations for the conservation of absorbent mass and solution energy [31] in any point of an expanding sheet are (respectively) expressed in polar coordinates (Fig. 2) as follows:

$$
\begin{array}{ll}
\frac{1}{r} \frac{\partial}{\partial r}\left(r V_{r} X\right)+\frac{\partial}{\partial z}\left(V_{z} X\right) & D \frac{\partial^{2} X}{\partial z^{2}} \\
\frac{1}{r} \frac{\partial}{\partial r}\left(r V_{r} T\right)+\frac{\partial}{\partial z}\left(V_{z} T\right) & \alpha \frac{\partial^{2} T}{\partial z^{2}}
\end{array}
$$

where $X$ and $T$ are the local mass fraction of absorbent and the solu tion temperature in the liquid sheet.

Because of the symmetry of the problem, Eqs. (3) and (4) are solved in a domain comprising only half the volume of the liquid sheet. The boundary conditions referring to this domain are pre sented in the following lines.

(i) The absorbent mass fraction and solution temperature at the initial section of the expanding sheet are:

$$
\begin{aligned}
& X\left(r_{0}, z\right) \quad \begin{cases}X_{0}, & 0 \leq z<\frac{\varepsilon_{0}}{2} \\
X_{s t}, & z \quad \frac{\varepsilon_{0}}{2}\end{cases} \\
& T\left(r_{0}, z\right) \quad \begin{cases}T_{0}, & 0 \leq z<\frac{\varepsilon_{0}}{2} \\
T_{s t}, & z \quad \frac{\varepsilon_{0}}{2}\end{cases}
\end{aligned}
$$

Here $\varepsilon_{0} \quad \varepsilon\left(r_{0}\right)$ is the thickness of the sheet at the initial posi tion. As in the work of Brauner [32], initial values of $X$ and $T$ at the surface have been selected equal to the values pro vided by the boundary layer theory (Section 5.4) near the ini tial position:

$$
\begin{array}{ll}
X_{s t} & X_{0} \frac{L_{a b s} c_{1}+X_{e q}\left(T_{0}\right) c_{D} L e^{1 / 2}}{L_{a b s} c_{1}+x_{0} c_{D} L e^{1 / 2}} \\
T_{s t} & \frac{X_{s t} \quad c_{2}}{c_{1}}
\end{array}
$$

Note that, due to the parabolic character of Eqs. (3) and (4), results for $X$ and $T$ are weakly affected by their initial values at the sheet surface. In fact, as will be confirmed later on, large Lewis number, $L e \gg 1$, causes rapid thermal dissipation as compared to mass transport. This avoids a substantial in crease of temperature at the surface of the sheet $z \quad \varepsilon_{0} / 2$ in $r \quad r_{0}$ and the temperature profile in Eq. (6) may be consid ered constant.

(ii) Gradients along the transversal direction are null at the plane of symmetry:

$$
\begin{array}{lll}
\left.\frac{\partial X}{\partial z}\right|_{z} & 0, & r \geq r_{0} \\
\left.\frac{\partial T}{\partial z}\right|_{z} \quad 0 & 0, \quad r \geq r_{0}
\end{array}
$$

(iii) The equilibrium mass fraction of absorbent at the free sur face, under small thermal variations, is commonly expressed (see for example, $[23,33]$ ) as a linear function of the solution temperature:

$X\left(r, z_{s}\right) \quad X_{e q}\left(T_{s}\right) \quad c_{1} \cdot T\left(r, z_{s}\right)+c_{2}$

where $z_{s} \quad \varepsilon / 2$ and $T_{s}$ are the values of the $z$ coordinate and temperature at the free surface, respectively. The constants $c_{1}$ and $c_{2}$ are properties of the mixture (Table 1) and depend on the absorbate vapour pressure $p_{v}$ which is assumed con stant throughout the chamber.

(iv) The absorbed mass and the heat of absorption released at the sheet surface are both transported by advection and diffusion into the fan sheet. An energy balance at the free surface of the fan sheet provides the next boundary condition [34]:

$\left.k \frac{\partial T}{\partial z}\right|_{s} \quad L_{a b s} \dot{m}_{v}^{\prime \prime}$

where $L_{a b s}$ is the specific heat of absorption of vapour in the solution and $\dot{m}_{v}^{\prime \prime}$ is the mass flux of absorbate vapour that is absorbed at the free surface of the expanding liquid sheet:

$\left.\dot{m}_{v}^{\prime \prime} \quad \frac{\rho D}{X_{s}} \frac{\partial X}{\partial z}\right|_{s}$

Advection at the sheet surface is due to the motion of the liquid solution relative to the free surface. Under the com 
mon operating conditions of absorption systems, Finite dilution at the interface (i.e. $X_{s}$ not close to unity) will make advective terms relevant [22]. Nakoryakov and co workers [35] also included advection in their model of absorption into a stagnant layer. Eq. (11) is equivalent to the expression deduced by inclusion of the net flow mo tion term (due in this case to absorbate diffusion) into Fick's law in a fixed coordinate reference system [36,32]. One should observe that, as radial diffusion terms are negli gible, the conservation equations ((3) and (4)) are parabolic and no boundary conditions are required downstream of the domain.

The following differential equation can be derived for the sheet thickness $\varepsilon$ from a mass balance in the fan sheet:

$\left.\frac{1}{r} \frac{d}{d r}\left(r \varepsilon \rho V_{0}\right) \quad \frac{2 D \rho}{X_{s}} \frac{\partial X}{\partial z}\right|_{s}$

where $\varepsilon_{0}$ is the initial condition of $\varepsilon$ in this equation. This last expression is coupled with the heat and mass balance equations for the sheet.

For convenience, the following transformations will be used for the radial and transversal coordinates, respectively:

$\hat{\xi} \quad \frac{1}{3 P e_{t} L e}\left(\begin{array}{ll}\xi^{3} & 1\end{array}\right)$

$\eta \frac{z}{z_{s}} \quad \frac{2 z}{\varepsilon}$

where $\xi \quad r / r_{0}$ and $P e_{t}$ is the Peclet number for the initial transver sal velocity of the solution at the interface:

$\left.P e_{t} \quad \frac{\left|V_{z, s}\right| \cdot z_{s}}{\alpha}\right|_{r r_{0}} \frac{V_{0} \varepsilon_{0}^{2}}{4 r_{0} \alpha}$

The normalised variable $\hat{\xi}$ resembles the dimensionless radial dis tance used by Tamir and Rachmilev [18]. The transversal coordi nate has been normalised via Eq. (15) in order to obtain $\eta \quad 1$ at the free surface. This change of variables not only simplifies the conservation equations, but also converts the physical domain of the expanding sheet into a rectangular domain $(\hat{\xi} \geq 0,0 \leq \eta \leq 1)$.

Under the proposed transformation of coordinates, the differen tial equations ( 3 ), (4) and (13) for the coupled heat and mass trans fer during non isothermal absorption of vapour into an expanding sheet become:

$$
\begin{aligned}
& \frac{\partial \hat{X}}{\partial \hat{\xi}} \quad \frac{\eta}{\hat{\varepsilon}} \frac{d \hat{\varepsilon}}{d \hat{\xi}} \frac{\partial \hat{X}}{\partial \eta}+\frac{1}{\hat{\varepsilon}^{2}} \frac{\partial^{2} \hat{X}}{\partial \eta^{2}} \\
& \frac{\partial \hat{T}}{\partial \hat{\xi}} \quad \frac{\eta}{\hat{\varepsilon}} \frac{d \hat{\varepsilon}}{d \hat{\xi}} \frac{\partial \hat{T}}{\partial \eta}+\frac{L e}{\hat{\varepsilon}^{2}} \frac{\partial^{2} \hat{T}}{\partial \eta^{2}}
\end{aligned}
$$

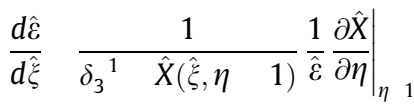

where $\hat{X} \quad\left(\begin{array}{lll}X & X_{0}\end{array}\right) /\left(X_{\infty} \quad X_{0}\right)$ and $\hat{T} \quad\left(\begin{array}{lll}T & T_{0}\end{array}\right) /\left(T_{\infty} \quad T_{0}\right)$ are the normalised temperature and mass fraction, and where $X_{\infty}$ and $T_{\infty}$ are the values reached under complete adiabatic saturation (see Appendix). $\hat{\varepsilon} \quad \varepsilon / \varepsilon_{N A}$ is the normalised sheet thickness, $\varepsilon_{N A}$ being the thickness of an expanding sheet that does not absorb vapour as it is not initially subcooled. Applying mass conservation, it is straightforward to demonstrate that $\varepsilon_{N A} \quad \varepsilon_{0} r_{0} / r$.

From the previous transformations, the boundary conditions of Eqs. (17) become:

$$
\begin{aligned}
& \hat{X}\left(\begin{array}{ll}
\hat{\xi} & 0, \eta
\end{array}\right) \quad\left\{\left.\begin{array}{lll}
0, & 0 \leq \eta<1 ; & \frac{\partial \hat{X}}{\partial \eta} \\
\hat{X}_{s t}, & \eta & 1
\end{array}\right|_{\eta} \quad 0, \quad \hat{\xi} \leq 0\right. \\
& \hat{T}\left(\begin{array}{ll}
\hat{\xi} & 0, \eta
\end{array}\right) \quad\left\{\left.\begin{array}{lll}
0, & 0 \leq \eta<1 ; & \frac{\partial \hat{T}}{\partial \eta}
\end{array}\right|_{\eta 0} \quad 0, \quad \hat{\xi} \leq 0\right. \\
& \hat{X}(\hat{\xi}, \eta \quad 1)+\delta_{2} \hat{T}(\hat{\xi}, \eta \quad 1) \quad 1+\delta_{2}, \quad \hat{\xi} \geq 0 \\
& \left.\left.L e \frac{\partial \hat{T}}{\partial \eta}\right|_{\eta 1} \frac{\delta_{1}}{\left.\delta_{2} X_{0} \cdot\left(\begin{array}{lll}
1 & \delta_{3} \cdot \hat{X}(\hat{\xi}, \eta & 1
\end{array}\right)\right)} \frac{\partial \hat{X}}{\partial \eta}\right|_{\eta 1} \\
& \hat{\varepsilon}(\hat{\xi} \quad 0) \quad 1
\end{aligned}
$$

where $\delta_{1}, \delta_{2}$ and $\delta_{3}$ are defined as:

$$
\begin{array}{ll}
\delta_{1} & \frac{c_{1} L_{a b s}}{C_{p}} \\
\delta_{2} & \frac{X_{\infty} X_{e q}\left(T_{0}\right)}{X_{0} X_{\infty}} \\
\delta_{3} & 1 \frac{X_{\infty}}{X_{0}} \frac{c_{1} \cdot \Delta T_{\text {sub }}}{X_{0} \cdot\left(1+\delta_{2}\right)}
\end{array}
$$

with $\Delta T_{\text {sub }} \quad T_{e q}\left(X_{0}\right) \quad T_{0}$ the initial subcooling of a sheet flow with $T_{e q}\left(X_{0}\right) \quad\left(\begin{array}{ll}X_{0} & c_{2}\end{array}\right) / c_{1}$. Substituting $X_{\infty}$ from (A.3) into the Eqs. (20) and (21), it is easy to find that:

$\delta_{2} \quad \frac{\delta_{1}}{X_{0}}$

Taking into account Eq. (22), the normalised expanding sheet Eqs. (17) and (18) only contain three dimensionless groups: the Le wis number $L e$, the dimensionless heat of absorption $\delta_{2}$ and the normalised subcooling $\delta_{3}$. Notice that these sheet equations form a nonlinear system for which no evident analytical solution exists. The sources of this nonlinearity are present in Eqs. (17) and (18.d): the term reflecting the mass flow rate increase and the term describing the surface advective velocity.

\subsection{Slowly increasing mass flow rate $(\dot{m} \approx$ const.) model}

Subcooling in refrigeration is commonly conducent to $\delta_{3} \ll 1$. This immediately gives, $d \hat{\varepsilon} / d \xi \ll 1$, which means that the sheet mass flow rate $\dot{m} \pi \varepsilon r \theta \rho V_{0} / 180$ increases slowly along radial direction. For sufficiently small values of $\delta_{3}$, it is possible to sim plify the nonlinear equations (17) in order to obtain the following linear system for the coupled heat and mass transfer in an expand ing sheet:

$$
\begin{array}{ll}
\frac{\partial \hat{X}}{\partial \hat{\xi}} & \frac{\partial^{2} \hat{X}}{\partial \eta^{2}} \\
\frac{\partial \hat{T}}{\partial \hat{\xi}} & L e \frac{\partial^{2} \hat{X}}{\partial \eta}
\end{array}
$$

Boundary conditions for this slowly increasing mass flow rate mod el are still defined by Eqs. (18), except for Eq. (18.d) which collapses to:

$\left.\left.L e \frac{\partial \hat{T}}{\partial \eta}\right|_{\eta \quad 1} \quad \frac{\partial \hat{X}}{\partial \eta}\right|_{\eta 1}$

It can be shown that the above linearised system of equations is equivalent to the expressions obtained using a simplified model that considers infinite dilution at the interface (e.g. null advective transport at the surface) in which $L_{a b s}$ is substituted by a modified specific heat of absorption $L_{a b s}^{*} \quad L_{a b s} / X_{0}$. Observe that the linearity of the Eqs. (23) and (24) simplifies their integration. 


\subsection{Large Lewis (Le $\gg 1$ ) model}

The Lewis number, $L e$, is much greater than one in most of the mixtures used in absorption systems. This means that diffusion heat transfer is more effective than mass transfer along the transversal direction. As a result $\hat{T}$ will only show a relevant dependence on $\hat{\xi}$. Under this simplification, Eq. (23.b) is no longer useful and the fol lowing energy balance is adopted in the whole section of the sheet:

$\frac{d(\dot{m} h)}{d r} \quad 2 \frac{\pi}{180} \theta \dot{m}_{v}^{\prime \prime} h_{v}$

Here, $h \quad C_{p}\left(T \quad T_{\text {ref }}\right)$ is the specific enthalpy of the liquid solution, taking $T_{\text {ref }}$ as a reference temperature.

Subsequently, under the approximation of large Lewis number and considering $\dot{m} \approx$ const., the system of equations to be solved is composed of the rearranged Eqs. (25) and (18.c),

$\left.\left.\frac{\partial \hat{X}}{\partial \eta}\right|_{\eta \quad 1} \quad \frac{1}{\delta_{2}} \frac{\partial \hat{X}}{\partial \hat{\xi}}\right|_{\eta \quad 1}$

$\hat{T}(\hat{\xi}) \quad \frac{1}{\delta_{2}} \hat{X}(\hat{\xi}, \eta \quad 1)+\frac{1}{\delta_{2}}+1$

along with Eq. (23.a) and the initial and symmetry boundary condi tions for $\hat{X}$, Eq. (18.a). Note that, when $L e \gg 1$, the initial conditions at the free surface collapses to $\hat{X}(0, \eta \quad 1) \quad 1+\delta_{2}$ and $\hat{T}(0, \eta \quad 1) \quad 0$. The main advantage of this system of equations for $L e \gg 1$ is that their analytical solution is greatly simplified. This is especially true as the normalised mass fraction $\hat{X}$ can be solved first and then introduced in Eq. (26.b) to calculate the normalised temperature $\hat{T}$.

\subsection{Boundary layer model}

A boundary layer model can be chosen to describe the initial re gion in which changes of temperature and species mass fraction in the expanding sheet only occur in a thin layer, close to the free sur face. The coupled heat and mass transport in the boundary layers can be modelled as if they were in a semi infinite volume of liquid, for instance comprising $\infty<\eta<1$ and $\hat{\xi} \geq 0$, as the boundary layers from opposite sheet surfaces do not touch each other. As will be shown in the simulation results, in this region $\hat{X}$ and $\hat{T}$ at the free surface stabilise to equilibrium values, $\hat{X}_{s t}$ and $\hat{T}_{s t}$, in the same manner as is found in falling film absorption [21]. A closed form solution to the system of Eqs. (23) in such a semi infinite domain can be obtained by means of the following self similar variable [31]:

$\phi \quad \frac{1 \eta}{2 \hat{\xi}^{1 / 2}}$

The transformed equations using (27) solely depend on $\phi$ and their integration reduces to expressions that depend linearly on the error function, $\operatorname{erf}(x) \quad 2 \pi^{1 / 2} \int_{0}^{x} e^{u^{2}} d u$ :

$\hat{X}(\phi) \quad \hat{X}_{s t} \cdot[1 \quad \operatorname{erf}(\phi)]$

$\hat{T}(\phi) \quad \hat{T}_{s t} \cdot\left[1 \quad \operatorname{erf}\left(\phi / L e^{1 / 2}\right)\right]$

where the stable values of mass fraction and temperature at the free surfaces are:

$$
\begin{array}{ll}
\hat{X}_{s t} & \frac{L e^{1 / 2} \cdot\left(\delta_{2}+1\right)}{\delta_{2}+L e^{1 / 2}} \\
\hat{T}_{s t} & \frac{\delta_{2}+1}{\delta_{2}+L e^{1 / 2}}
\end{array}
$$

Eqs. (28) and (29) are almost identical to the expressions first ob tained by Nakoryakov and Grigorieva [37] regarding absorption into a falling film over an isothermal wall. Though similar, the expres sions for $\hat{T}_{s t}$ or $\hat{X}_{s t}$ in the present investigation and in [37] differ, as no advective velocity relative to the interface was included in [37].

The point $\hat{\xi}_{\text {tr }}$, where a transition starts between boundary layer and fully developed flow in the sheet, can be roughly estimated as the radial position wherein temperature at the symmetry plane of the sheet increases to a sensible fraction $c_{k}$ of $\hat{T}_{s t}$ after the merger of opposing thermal boundary layers:

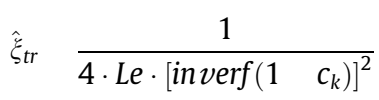

with inverf, the inverse function of the error function, and $0<c_{k}<1$.

\section{Solving procedure}

\subsection{Numerical solution of the nonlinear model}

A finite difference numerical method [38] has been developed for the solution of the set of parabolic differential equations (17) together with their boundary conditions (18). It consists of a semi implicit numerical scheme that marches the solution along the radial direction of the sheet from the initial position $r \quad r_{0}$. A detailed description of this method can be found in [34]. Since the equations involved in the problem are nonlinear, their numer ical solution is computationally demanding inasmuch as it requires the inversion of a large matrix during every integration step. The numerical scheme has been programmed in a personal computer and the accuracy of the results has been assessed through the sen sitivity of the calculated $\hat{X}$ and $\hat{T}$ on the mesh size, as well as the concordance between global energy and mass balances in the sheet.

Table 2 contains geometric and hydrodynamic parameters that were selected for the simulated reference cases. Their values have been obtained from a standard flat fan spray nozzle whose exit section has $d_{e q}=2 \mathrm{~mm}$ equivalent diameter and $a_{0}=9.3 \mathrm{~mm}$ width, operating at an injection pressure between 1.0 and 2.0 bar.

\subsection{Analytical and numerical solution of the $\dot{m} \approx$ const. model}

The assumption of slowly increasing mass rate (described ear lier) leads to a simplified set of linear differential equations (Eqs. (23), (18.a c) and (24)). Thus, it is possible to follow a procedure analogous to the one first presented by Nakoryakov and Grigorieva [39] on falling films in order to obtain a series solution of $\hat{X}$ and $\hat{T}$. An interesting result of the series solution of $\hat{X}$ and $\hat{T}$ is that it de pends on the integral values of mass fraction and temperature at the initial position ( $\left.\begin{array}{ll}\hat{\xi} & 0\end{array}\right)$ but not on the specific values of $\hat{X}$ and $\hat{T}$ at the free surface $\left(\begin{array}{lll}\hat{\xi} & 0, \eta & 1\end{array}\right)$. In practice, obtaining the eigen values of the series becomes cumbersome while the resulting solu tion converges quite slowly. It is also found that the series solution values for $\hat{X}$ and $\hat{T}$ ripple near the initial position of the expanding sheet on account of numerical artifacts produced by the sharp boundary condition at $\hat{\xi} \quad 0$. Similar effects have been also re ported in $[33,40]$. Therefore, all the results presented in this study under the $\dot{m} \approx$ const. approximation have been integrated numer

Table 2

Reference values of sheet geometry and operation.

\begin{tabular}{llll}
\hline$\varepsilon_{0}[\mathrm{~m}]$ & $r_{0}[\mathrm{~m}]$ & $\theta\left[{ }^{\circ}\right]$ & $V_{0}\left[\mathrm{~m} \mathrm{~s}^{1}\right]$ \\
\hline $3.38 \times 10^{4}$ & $7.64 \times 10^{3}$ & 75 & 6 \\
\hline
\end{tabular}


Table 3

Relevant dimensionless groups.

\begin{tabular}{lll}
\hline & $\mathrm{LiBr}-\mathrm{H}_{2} \mathrm{O}$ & $\mathrm{LiNO}_{3}-\mathrm{NH}_{3}$ \\
\hline $\mathrm{Le}$ & 88.8 & 686 \\
$\mathrm{Pe} e_{t}$ & 164 & 44.2 \\
$\delta_{1}$ & 6.45 & 1.86 \\
$\delta_{2}$ & 10.2 & 2.95 \\
$\delta_{3}$ & $7.62 \times 10^{3}$ & $2.01 \times 10^{2}$ \\
\hline
\end{tabular}

ically via the algorithm developed for the nonlinear model after imposing $\delta_{3} \quad 0$.

\subsection{Analytical solution of the Le $\gg 1$ model}

In this case, the series solutions chosen for the normalised mass fraction and temperature in the expanding sheet consist of:

$\hat{X}(\hat{\xi}, \eta) \quad C_{0}+\sum_{n 1}^{\infty} C_{n} Q_{n}(\eta) e^{\zeta_{n}^{2 \hat{\xi}}}$

$\hat{T}(\hat{\xi}) \quad D_{0}+\sum_{n 1}^{\infty} D_{n} e^{\zeta_{n}^{2} \hat{\xi}}$

where $Q_{n}(\eta) \quad \cos \left(\zeta_{n} \eta\right)$. The first terms of the series (31) are $C_{0}=1$ and $D_{0} \quad 1$ in order to assure that the normalised mass fraction and temperature tends to one as $\hat{\xi} \rightarrow \infty$. The remaining coefficients are [34]:

$C_{n} \frac{2 \cdot\left(1+\delta_{2}\right) \cdot \cos \left(\zeta_{n}\right)}{\delta_{2}+\cos ^{2}\left(\zeta_{n}\right)}$

$D_{n} \frac{2\left(1+\delta_{2}\right)}{\delta_{2}\left(1+\delta_{2}\right)+\zeta_{n}^{2}}$

The eigenvalue $\zeta_{n}$ should satisfy the transcendental equation,

$\tan \left(\zeta_{n}\right) \quad \frac{\zeta_{n}}{\delta_{2}}$

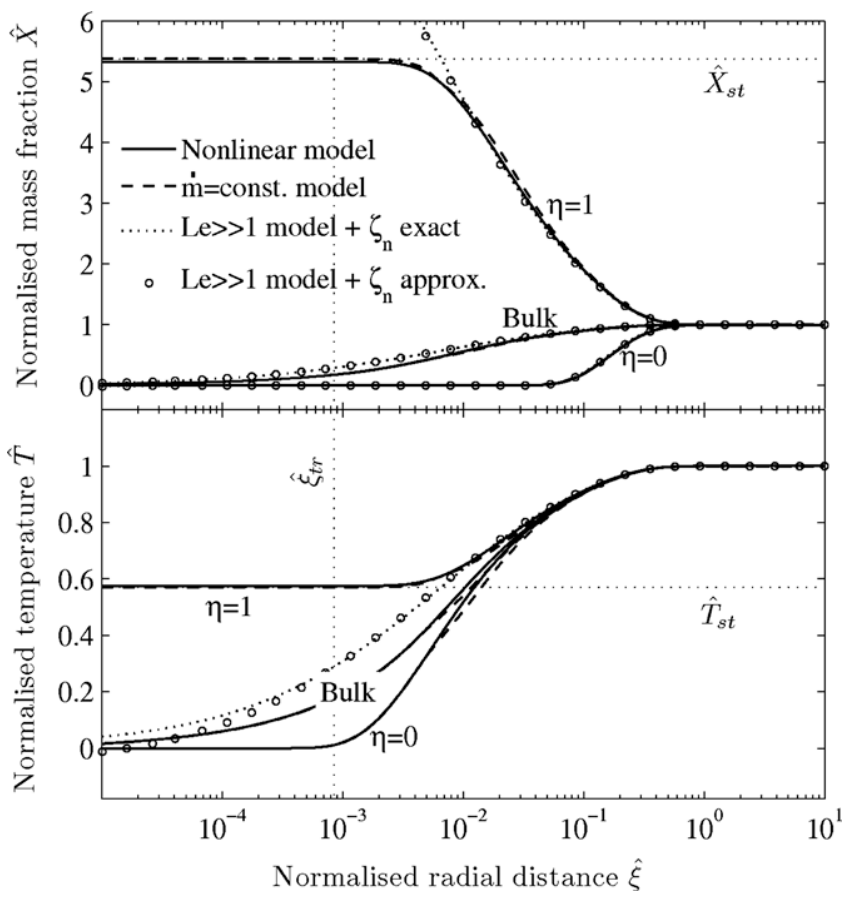

Fig. 3. Radial variation of mass fraction and temperature in the expanding sheet for the pair $\mathrm{LiBr}-\mathrm{H}_{2} \mathrm{O}$ : local values at two transversal positions, $\eta$, and transversally averaged (or bulk) results.

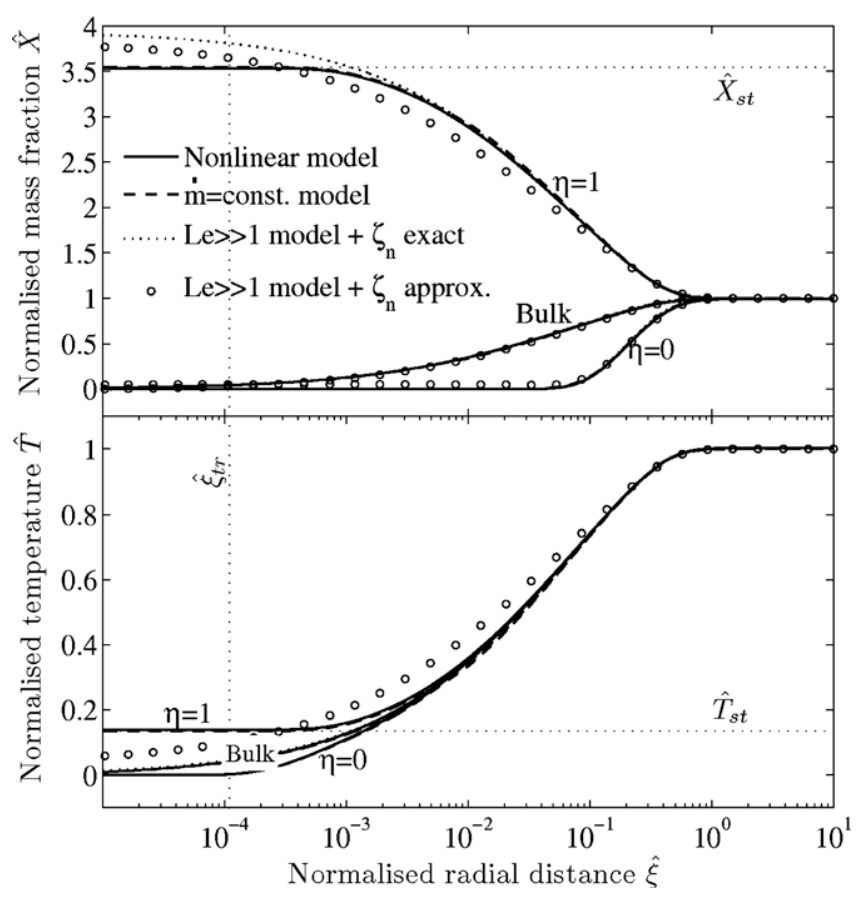

Fig. 4. Radial variation of mass fraction and temperature in the expanding sheet for the pair $\mathrm{LiNO}_{3}-\mathrm{NH}_{3}$ : local values at two transversal positions, $\eta$, and transversally averaged (or bulk) results.

which has been obtained by requiring the series (31) to satisfy the boundary condition (26.a). An explicit equation for the estimation of $\zeta_{n}$ is proposed in the present work:

$\zeta_{n} \approx \frac{\delta_{2} \pi}{8 n} \cdot\left(1 \quad \sqrt{1+\left(\frac{4 n}{\delta_{2}}\right)^{2}}\right)+n \pi, \quad n \geq 1$

This equation, which is deduced in approximating the tangent func tion by a rational expression of second order, will be assessed as an alternative of the exact solution of Eq. (33). The accuracy of the eigenvalues estimated using (34) rapidly increases with $n$ and with $\delta_{2}$, and is good enough for the calculation of $\hat{X}$ and $\hat{T}$ in practical engineering applications. For example, taking $\delta_{2}$ values from Table 3 , the largest error in accuracy appears in $\zeta_{1}$ and is less than $0.8 \%$ and $4.2 \%$ of the exact values that satisfy Eq. (33) in sheets of $\mathrm{LiBr}$ $\mathrm{H}_{2} \mathrm{O}$ and $\mathrm{LiNO}_{3} \mathrm{NH}_{3}$, respectively.

\section{Results and discussion}

\subsection{Temperature and mass fraction profiles}

Figs. 3 and 4 contain the stream wise computed profiles of nor malised mass fraction $\hat{X}$ and temperature $\hat{T}$ for the reference pairs $\mathrm{LiBr} \mathrm{H}_{2} \mathrm{O}$ and $\mathrm{LiNO}_{3} \mathrm{NH}_{3}$. The plotted results correspond to the four models analysed in this study.

Relevant dimensionless groups used in the simulation can be found in Table 3 based on the operative conditions of Table 2 and the properties of Table 1 . Values at the free surface $\eta 1$, at the centre of the sheet $\eta \quad 0$ (i.e. the plane of symmetry) and at points in the bulk have been represented in the curves. Bulk values correspond to the normalised transversely averaged mass fraction and temperature:

$\hat{X}_{b}(\hat{\xi}) \quad \frac{X_{b} \quad X_{0}}{X_{\infty}} \quad X_{0} \quad \int_{0}^{1} \hat{X}(\hat{\xi}, \eta) d \eta ; \quad \hat{T}_{b}(\hat{\xi}) \quad \int_{0}^{1} \hat{T}(\hat{\xi}, \eta) d \eta$ 
Table 4

Main results.

\begin{tabular}{lll}
\hline & $\mathrm{LiBr}-\mathrm{H}_{2} \mathrm{O}$ & $\mathrm{LiNO}_{3}-\mathrm{NH}_{3}$ \\
\hline$\hat{\xi}_{t r}$ & $8.49 \times 10^{4}$ & $1.10 \times 10^{4}$ \\
$\hat{X}_{s t}$ & 5.38 & 3.55 \\
$\hat{T}_{s t}$ & 0.571 & 0.135 \\
$S h_{\infty}$ & 1.15 & 2.25 \\
$\overline{S h}_{\infty}$ & 3.19 & 3.34 \\
$\max \left(\frac{m_{v}}{m_{0}}\right)$ & $7.68 \times 10^{3}$ & $2.05 \times 10^{2}$ \\
\hline
\end{tabular}

The normalised bulk mass fraction is equivalent to the "Approach to Equilibrium Factor F" [41] when specialised to the case of adiabatic absorption [42], and can be viewed as an efficiency of the absorp tion process.

Three main regions of flow behaviour are clearly distinguished in the results of the complete nonlinear model: a boundary layer, a transition and a fully developed region.

\subsubsection{Boundary layer region}

In this initial portion of the sheet, the absorbate mass and the heat transported into the sheet affect only a thin layer close to the free surface. The remaining volume of the sheet is still at the initial temperature and mass fraction conditions. As Figs. 3 and 4 show, this region ranges from the initial position $\hat{\xi} 0$, to approximately the normalised transition radius $\hat{\xi}_{\text {tr }}$ (see Eq. (30)), which has been calculated with $c_{k} \quad 0.01$ and included in Table 4. Observe that the boundary layer region is longer for the $\mathrm{LiBr} \mathrm{H}_{2} \mathrm{O}$ solution than it is for the $\mathrm{LiNO}_{3} \mathrm{NH}_{3}$ solution be cause the thermal boundary layer reaches the sheet symmetry plane earlier in the ammonia solution (on account of its larger Le wis number).

Normalised absorbent mass fraction $\hat{X}$ and solution tempera ture $\hat{T}$ stabilise at the free surface of the boundary layer region according to the results of the complete nonlinear model. These local stable values are well predicted by $\hat{X}_{s t}$ and $\hat{T}_{s t}$ in the boundary layer approximation theory. The fact that $\hat{X}$ and $\hat{T}$ become stable at the free surface is due to the counteracting effects of mass absorp tion (which tends to increase solution temperature) and species equilibrium at the surface (which tends to decrease the solution temperature whenever the absorbate mass fraction increases). It should be noticed, however, that the parabolic set of equations (3) and (4), and subsequent models, cease to be valid in a previous region, very close to $r_{0}$, because diffusion along the radial coordi nate is not negligible compared to transversal diffusion when $\hat{\xi} \rightarrow 0$. Nevertheless, the contribution of this early region to the to tal amount of heat and mass transferred into the sheet is negligible since its exposed surface to the vapour is extremely small. This la ter fact also explains why the solution is weakly affected by the selection of the initial conditions at the sheet free surface $\left(\begin{array}{ll}\eta & 1\end{array}\right)$ performed in Eqs. (5) and (6).

The transversal profiles of normalised mass fraction and temperature are depicted in Figs. 5 and 6 . The predicted results from the boundary layer model and from the numerical simula tion of the entire sheet match almost perfectly inside the boundary layer region, bearing out the correctness of this approximation.

In the boundary layer region, as well as in the rest of the sheet, a numerical solution using the $\dot{m}$ const. model gives results that are practically indistinguishable from the solution of the complete nonlinear model because common subcooling in adiabatic absorp tion, of the order of magnitude of $10^{\circ} \mathrm{C}$, is conducent to very small $\delta_{3}$ (see Table 3). By definition, the approximate model for $L e \gg 1$ cannot provide thermal gradients in the transversal direction and hence fails in predicting the normalised mass fraction at the free surface of the sheet. For clarity, curves of this model have not been included in Figs. 5 and 6 for $\xi<\hat{\xi}_{t r}$. Notice that a decrease in Le in creases the thermal gradient and reduces the transversal mass fraction gradient in the boundary layer region, since $\hat{T}_{s t}$ decreases while $\hat{X}_{s t}$ diminishes.

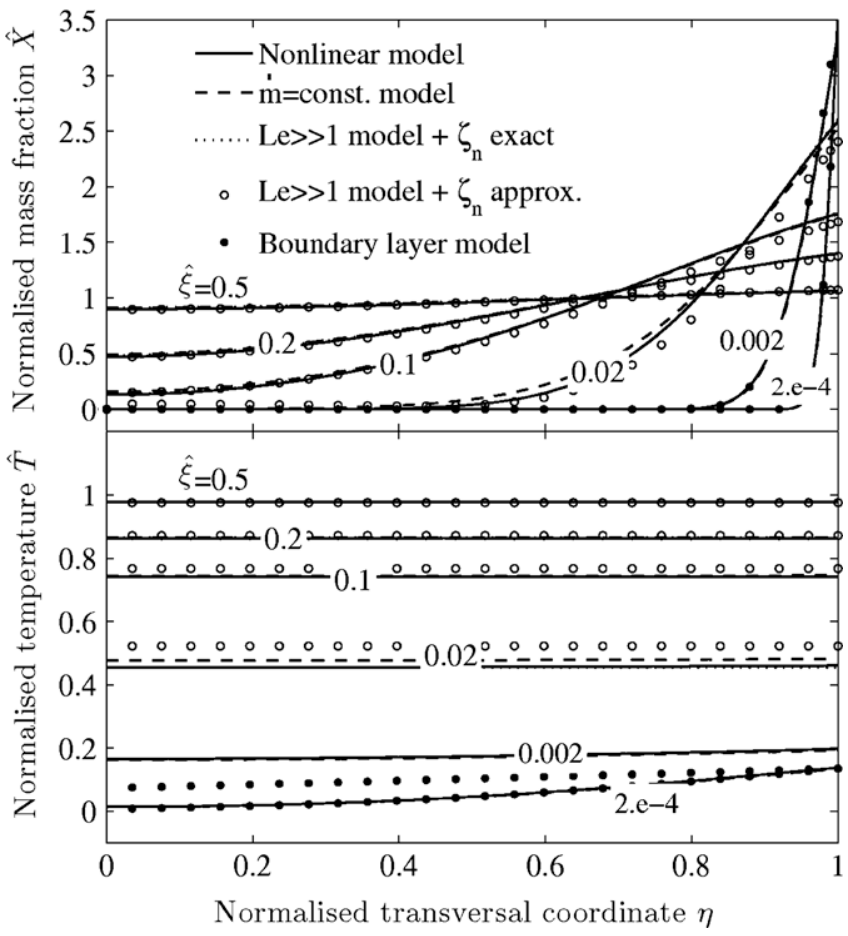

Fig. 6. Transversal profiles of mass fraction and temperature in the expanding sheet for the pair $\mathrm{LiNO}_{3}-\mathrm{NH}_{3}$ at various normalised radial distances $\hat{\xi}$.
Fig. 5. Transversal profiles of mass fraction and temperature in the expanding sheet for the pair $\mathrm{LiBr}-\mathrm{H}_{2} \mathrm{O}$ at various normalised radial distances $\hat{\xi}$. 


\subsubsection{Transition region}

A transition region separates the boundary layer behaviour from the final fully developed heat and mass transfer region in the expanding sheet. The transition starts when the temperature boundary layers reach the symmetry plane of the sheet at $\hat{\xi}_{t r}$. From this moment, a change of morphology in the temperature and mass fraction profiles occurs, causing the loss of stable conditions for $\hat{T}$ and $\hat{X}$ at the free surface of the sheet. Numerical solutions of the nonlinear model as well as the $\dot{m}$ const. model in Figs. 36 lend evidence to this fact. The transition region can be considered nearly completed when absorbate mass fraction at the centre of the sheet starts to increase. To be precise, heat and mass transfer in the expanding sheet are not near fully developed until $\xi \approx 0.2$, as will be shown later.

Of course it is clear that notorious discrepancies between the nonlinear model and the boundary layer approximation arise in the transition region. This mismatch is shown here in Fig. 6 for $\eta \quad 0.002$. Using the Le $\gg 1$ model and the exact calculation of $\zeta_{n}$ (Eq. (33)), acceptable results can be obtained in this region for the normalised mass fraction profiles at the sheet surface. This is especially true for the $\mathrm{LiNO}_{3} \mathrm{NH}_{3}$ solution. In this working pair, the Lewis number is relatively large so that the transversal gradi ent in temperature is less pronounced than in $\mathrm{LiBr} \mathrm{H}_{2} \mathrm{O}$. In fact the actual transversal temperature profiles in the $\mathrm{LiNO}_{3} \mathrm{NH}_{3}$ solu tion are practically horizontal within this region, yielding quite accurate predictions of $\hat{T}$. In the case of the ammonia solution how ever, the explicit approximation of $\zeta_{n}$ provided by Eq. (34) (hollow circles in the plot) leads to a reduction in the accuracy of the re sults as $\delta_{2}$ for this working pair is lower than it is in the aqueous solution. As the analytical solutions for the $L e \gg 1$ model demon strates, $\hat{X}_{b}$ tends to $\hat{T}_{b}$ when Le increases.

\subsubsection{Fully developed flow region}

After the merger of mass fraction boundary layers, the flow can be regarded as fully developed, in the sense that heat and mass transfer are commanded by the bulk temperature and mass frac tion of the flow and not by the shape evolution of their gradients in the sheet. $\hat{X}$ and $\hat{T}$ tend to unity when $\hat{\xi} \rightarrow \infty$ as described in Appendix A. Transversal profiles given in Figs. 5 and 6 show that, for a sufficiently large streamwise distance, transversal gradients of temperature and absorbent mass fraction diminish and their quantities at the surface and at the symmetry plane of the sheet tend to equality with their bulk values. Henceforth, it is in the fully developed zone where the $L e \gg 1$ model, taking $\zeta_{n}$ either exact or approximate, delivers results in very fine agreement with the non linear model.

It should be mentioned that the profiles of normalised mass fraction and temperature, described here for expanding sheets, posses a distinctive shape similar to the case of falling film absorp tion described in $[33,32,37,43]$.

\subsection{Sheet thickness distribution}

Fig. 7 contains the normalised thickness profiles along the streamwise direction for the two solution pairs selected in this investigation. $\hat{\varepsilon}$ increases owing to the continuous incorporation of absorbate vapour into the solution during absorption. Indirect calculation of the normalised thickness $\hat{\varepsilon}$ has also been performed knowing that the sheet thickness is directly proportional to the sheet's mass flow rate:

$\hat{\varepsilon} \quad \frac{\dot{m}}{\dot{m}_{0}} \frac{1}{1 \delta_{3} \hat{X}_{b}}$

where $\dot{m}_{0} \quad \pi \varepsilon_{0} r_{0} \theta \rho V_{0} / 180$ is the mass flow rate of solution at the initial position in the sheet and $\hat{X}_{b}$ is the normalised bulk mass frac

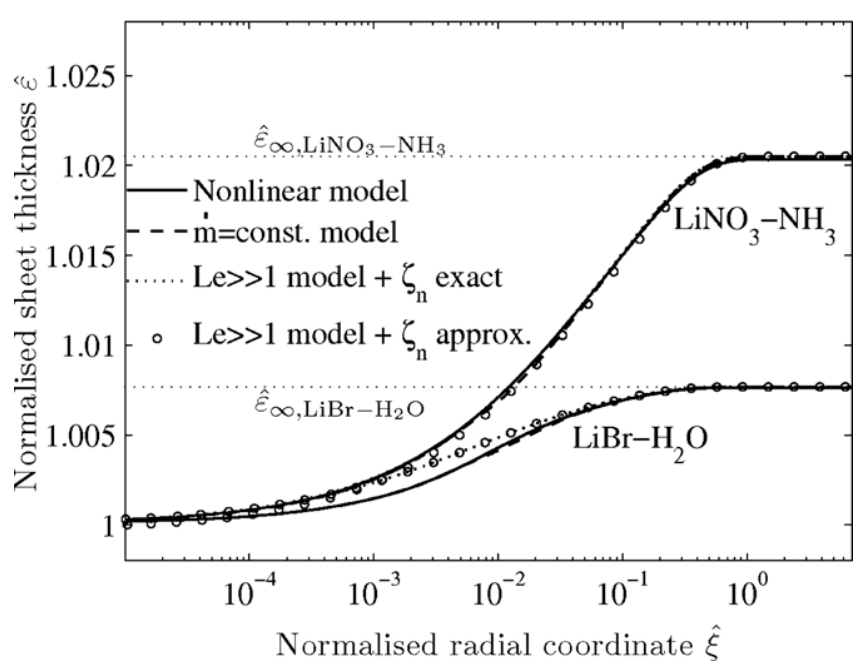

Fig. 7. Radial variation of the normalised sheet thickness for the pairs $\mathrm{LiBr}-\mathrm{H}_{2} \mathrm{O}$ and $\mathrm{LiNO}_{3}-\mathrm{NH}_{3}$.

tion, Eq. (35). Excellent agreement is encountered in Fig. 7 between $\hat{\varepsilon}$ as calculated directly with the nonlinear model and $\hat{\varepsilon}$ indirectly estimated (through Eq. (36)) using the results of the $\dot{m}$ const. model. As expected, the $L e \gg 1$ model presents poorer quantitative results in the boundary layer region for the solution with lower Lewis number value $\left(\mathrm{LiBr} \mathrm{H}_{2} \mathrm{O}\right)$. Differences between the results calculated with exact $\zeta_{1}$ and approximate $\zeta_{1}$ are not relevant when calculating the sheet thickness. As will be shown later, this is also true for the Sherwood numbers.

Fig. 7 demonstrates that Eq. (36) can be used to obtain accurate estimation of the maximum normalised thickness attainable under complete adiabatic saturation of the sheet when $\hat{\xi} \rightarrow \infty$; that is, when $\hat{X}_{b} \quad 1$ :

$\hat{\varepsilon}_{\infty} \frac{1}{1 \delta_{3}}$

Under the reference parameters of Table 3, the normalised thick ness grows faster with $\hat{\xi}$ and reaches the larger $\hat{\varepsilon}_{\infty}$ (the largest absorbate absorption per unit of initial solution flow rate) in sheets of $\mathrm{LiNO}_{3} \quad \mathrm{NH}_{3}$ solution than in sheets of $\mathrm{LiBr} \mathrm{H}_{2} \mathrm{O}$.

\subsection{Local and mean mass transfer coefficients}

In order to characterise the mass transfer coefficients, the local Sherwood number, $S h$, and the mean Sherwood number, $\overline{S h}$, of the sheet can be calculated using as characteristic lengths the sheet half thickness, $L_{c} \quad \varepsilon / 2$, and the sheet mean half thickness (sheet volume/free surface area), $\bar{L}_{c} \quad \varepsilon_{0} /(\xi+1)$, respectively:

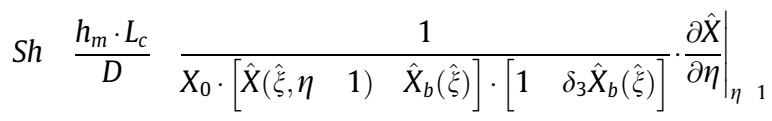

$$
\begin{aligned}
& \left.\overline{S h} \frac{h_{m} L_{c}}{D} \frac{4 P e_{t} L e \hat{X}_{b}}{\left(\xi^{2}\right.} 1\right) \cdot(\xi+1) \cdot X_{0} \cdot\left(\begin{array}{ll}
1 & \left.\delta_{3} \hat{X}_{b}\right) \cdot \Delta \hat{X}_{l m}
\end{array}\right.
\end{aligned}
$$

where $\left.h_{m} \quad \dot{m}_{v}^{\prime \prime} /\left[\begin{array}{ll}\rho\left(X_{b}\right. & X_{s}\end{array}\right)\right]$ is the local heat transfer coefficient [31] at $\hat{\xi} . \bar{h}_{m} \quad \dot{m}_{v} /\left[\rho A_{s} \Delta X_{l m}\right]$ is the mean mass transfer coefficient [44], which represents a mean value of $h_{m}$ throughout the sheet surface $A_{s} \pi \theta\left(r^{2} \quad r_{0}^{2}\right) / 180$ up to a given $\hat{\xi}$. The logarithmic mean absor bate mass fraction difference $\Delta X_{l m} \quad\left(\Delta X_{0} \quad \Delta X\right) / \ln \left(\Delta X_{0} / \Delta X\right)$ has been included in the $\bar{h}_{m}$ definition, $\Delta X \quad X_{b} \quad X_{s}$ being the differ ence between bulk and surface absorbent mass fractions at $\hat{\xi}\left(\Delta X_{0}\right.$ is the same difference evaluated at $\hat{\xi} \quad$ 0) [42]. In Eq. (38.b) $\Delta \hat{X}_{l m}$ is calculated with $\Delta \hat{X} \quad \hat{X}_{s} \quad \hat{X}_{b}$. 


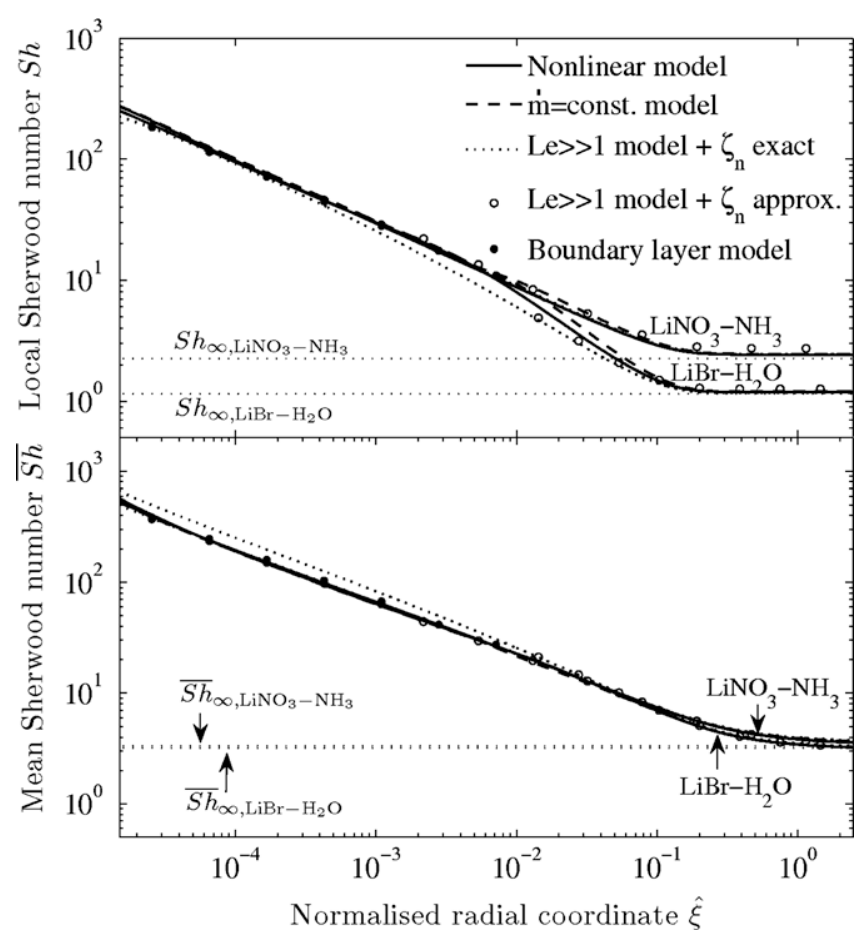

Fig. 8. Radial variation of the local and mean Sherwood numbers in the expanding sheet for the pairs $\mathrm{LiBr}-\mathrm{H}_{2} \mathrm{O}$ and $\mathrm{LiNO}_{3}-\mathrm{NH}_{3}$.

Fig. 8 depicts the local and mean Sherwood numbers for the two working pairs under study. Progressive relaxation of the absorbate mass fraction gradient into the sheet monotonically decreases the local Sherwood number from infinite at $\hat{\xi} \quad 0$ toward a constant asymptotic value $S h_{\infty}$ for $\hat{\xi} \rightarrow \infty$. The mean Sherwood number reproduces this tendency, having always a superior value than its local definition, because very large values of local $h_{m}$ at initial ra dial positions significantly increase $\bar{h}_{m}$. In general the $\dot{m}$ const. model provides almost identical results to the full nonlinear model. Although in qualitative agreement, the $L e \gg 1$ analytical model shows discrepancies in the boundary layer region for the solution with the smaller $\mathrm{Le}$ (i.e. $\mathrm{LiBr} \mathrm{H}_{2} \mathrm{O}$ ). It is also possible to explicitly estimate the Sherwood number in the boundary layer region by substituting Eqs. (28) into (38):

$$
\begin{aligned}
& S h_{B L} \frac{1}{X_{0} \cdot\left(\begin{array}{ll}
1 & \delta_{3} \hat{X}_{s t}
\end{array}\right) \cdot \sqrt{\pi \hat{\xi}}} \approx \frac{1}{X_{0} \cdot \sqrt{\pi \hat{\xi}}} \\
& \overline{S h}_{B L} \frac{8\left[\mathrm{Pe}_{t} \operatorname{Le}\left(\begin{array}{ll}
\xi^{3} & 1
\end{array}\right) / 3 \pi\right]^{1 / 2} \hat{X}_{s t}}{X_{0} \cdot\left(\begin{array}{ll}
1 & \delta_{3} \hat{X}_{s t}
\end{array}\right) \cdot\left(\begin{array}{ll}
\xi^{2} & 1
\end{array}\right) \cdot(\xi+1) \cdot \Delta \hat{X}_{l m}} \\
& \approx \frac{8 P e_{t} L e \hat{\xi}^{1 / 2} \hat{X}_{s t}}{X_{0} \cdot \sqrt{ } \pi \cdot\left(\xi^{2} 1\right) \cdot(\xi+1) \cdot \Delta \hat{X}_{l m}}
\end{aligned}
$$

Here, Eq. (28.a) has been used to obtain the normalised bulk mass fraction $\hat{X}_{b} 2 \hat{X}_{s t}(\hat{\xi} / \pi)^{1 / 2} /\left(\begin{array}{ll}1 & \delta_{3} \hat{X}_{s t}\end{array}\right)$ required in $\Delta \hat{X}_{l m}$. Notice that it is obtained a fine accordance between Eqs. (39) and the numerically obtained Sherwood number in the boundary layer re gion except, notably, for $\hat{\xi}$ very close to zero where numerical inte gration does not converge to boundary layer self similar solutions. This excellent agreement confirms the hypothesis that the numeri cally calculated transport of mass and heat is not substantial in the very early region, prior to the surface stabilisation to $\hat{X}_{s t}$ and $\hat{T}_{s t}$. Lo cal Sherwood numbers of the two working pairs exhibit practically coincident $S h_{B L}$ profiles as both solutions have identical $X_{0}$.

The existence of the asymptotic minimum Sherwood numbers $S h_{\infty}$ and $\overline{S h}_{\infty}$ is due to the full development of the thermal and mass fraction fields. In view of the local Sherwood profile, it can be said quite rigorously that the coupled heat and mass transfer do not approach fully developed conditions before $\hat{\xi} \approx 0.2$. Includ ing the results of the Le $\gg 1$ model, Eq. (31), into Eq. (38), these fully developed flow Sherwood numbers can be estimated for the limit $\hat{\xi} \rightarrow \infty$ :

$S h_{\infty} \approx \frac{\zeta_{1}^{2}}{X_{0} \cdot\left(1+\delta_{2}\right)}$

$\overline{S h}_{\infty} \approx \frac{4}{3}\left(\frac{\delta_{2}}{L e^{1 / 2}}+1\right) S h_{\infty}$

where $\zeta_{1}$ is the first eigenvalue, which can be approximated by Eq. (34) for $n=1$. According to Fig. 8, Eqs. (40) provide good quantita tive approximations in the fully developed region. Eqs. (40) also ex plain that the Sherwood number for the $\mathrm{LiNO}_{3} \mathrm{NH}_{3}$ solution is greater than that for $\mathrm{LiBr} \mathrm{H}_{2} \mathrm{O}$ solution because the first working pair has the highest $\delta_{2}$ ( $\zeta_{1}$ being weakly dependent on $\delta_{2}$ ).

\subsection{Effect of the aperture angle}

Normalised mass fraction, temperature and sheet thickness profiles along $\hat{\xi}$ are independent of the expanding sheet aperture angle $\theta$. This is because its effect is concealed in $\hat{\xi}$ through $P e_{t}$, which is a function of $r_{0} \quad a_{0} /[2 \sin (\theta / 2)]$. This is also true for $S h$ and it is almost true for $\overline{S h}$ on account of Eqs. (38). It can be demonstrated that exactly the same dimensionless equations as those deduced in this investigation for expanding sheets arise for non expanding sheets. This follows provided the next variables are used in non expanding sheets instead of $\hat{\xi}, \eta$ and $\hat{\varepsilon}$ :

$\hat{\xi}_{N E} \quad \frac{2 y}{P e_{l} L e \varepsilon_{0}}, \quad \eta_{N E} \quad \frac{2 z}{\varepsilon}, \quad \hat{\varepsilon}_{N E} \quad \frac{\varepsilon}{\varepsilon_{0}}$

where $y$ accounts for the streamwise coordinate $(y \quad 0$ at the initial section of the sheet), $\varepsilon_{0}$ is the initial thickness of the non expanding sheet and $P e_{l} \quad V_{0} \varepsilon_{0} / 2 \alpha$ is the Peclet number based on the stream wise velocity of the non expanding sheet. Note that, in expanding sheets, $y \quad r \quad r_{0}$. Hence, expanding and non expanding sheets, at a given $y$, each satisfy the following relation:

$\hat{\xi} \quad \hat{\xi}_{N E} \quad \frac{\left(P e_{t} L e\right)^{2}}{3} \cdot \hat{\xi}_{N E}^{3}+P e_{t} L e \cdot \hat{\xi}_{N E}^{2}$

and thus, $\hat{\xi} \quad \hat{\xi}_{N E}>0$. This means that (for similar initial cross sec tional area, equal injection velocity and same working pairs) an expanding sheet configuration of a given (dimensional) length, be haves just as a non expanding sheet of greater length. Table 5 re ports some examples of dimensionless sheet length $\psi y 2 / \varepsilon_{0}$ required for an approach to equilibrium factor of $F \quad 95 \%$ $\left(\begin{array}{ll}\hat{X}_{b} & 0.95\end{array}\right)$. Results are calculated with the nonlinear model and fixed $a_{0}$ from Table 2 . As $\theta$ decreases, the sheet length to reach the pre set $95 \%$ saturation increases. The largest $\psi$ takes place al ways for the ammonia solution because it has the larger product $\mathrm{Pe}$ Le. Using the nozzle geometry of Table 2, sheet lengths of 72 or $125 \mathrm{~mm}$ are required for $\mathrm{LiBr} \mathrm{H}_{2} \mathrm{O}$ or $\mathrm{LiNO}_{3} \mathrm{NH}_{3}$ solutions, respectively, in order to reach $F \quad 95 \%$. In general, the larger the an gle $\theta$, the greater $\hat{\xi} \quad \hat{\xi}_{N E}$ and in turn, expanding sheets have a great er performance advantage (higher $h_{m}$ and $A_{s}$ ) over non expanding

Table 5

Dimensionless length $\psi$ for $F \quad 95 \%$ and fixed $a_{0}$.

\begin{tabular}{lll}
\hline$\theta\left[{ }^{\circ}\right]$ & $\mathrm{LiBr}-\mathrm{H}_{2} \mathrm{O}$ & $\mathrm{LiNO}_{3}-\mathrm{NH}_{3}$ \\
\hline 0 & $1.18 \times 10^{5}$ & $5.50 \times 10^{5}$ \\
15 & 2290 & 3970 \\
75 & 852 & 1450 \\
150 & 631 & 1073 \\
\hline
\end{tabular}


sheets of similar length. Similar comments for a non expanding sheet apply to a falling film of initial thickness equal to $\varepsilon_{0} / 2$ flowing along an adiabatic wall when using a model that approximates the velocity field by a constant profile.

\subsection{Effect of the heat of absorption}

The heat of absorption has significant influence, through $\delta_{2}$ (see Eq. (22)), on the heat and mass transfer into the sheet. Fig. 9 shows the sensitivity to $\delta_{2}$ on the normalised bulk mass fraction $\hat{X}_{b}$ and on the sheet absorption rate $\dot{m}_{v} / \dot{m}_{0}$ for a solution possessing the same Le and the same dimensionless group $c_{1} \Delta T_{\text {sub }} / X_{0}$ than the $\mathrm{LiBr} \mathrm{H}_{2} \mathrm{O}$ solution (Table 1 ). For the sake of clarity, the Le $\gg 1$ model with approximate $\zeta_{1}$ has not been included in Fig. 9. This model repro duces the same trend as that seen in the $\hat{X}_{b}$ curves in Figs. 3 and 4. The rate of absorption can be related to $\hat{X}_{b}$ thanks to the follow ing relation:

$\frac{\dot{m}_{v}}{\dot{m}_{0}} \quad \hat{\varepsilon} \quad 1 \quad \frac{\delta_{3} \hat{X}_{b}}{1 \delta_{3} \hat{X}_{b}} \approx \delta_{3} \hat{X}_{b}$

where $\dot{m}_{v} \quad \dot{m} \quad \dot{m}_{0}$ is the mass flow of vapour absorbed into the sheet from $y \quad 0$ to a distance $\hat{\xi}$. An increase of $\delta_{2}$ significantly in creases $\hat{X}_{b}$ (Eq. (35)) for a given $\hat{\xi}$ since $X_{\infty}$ decreases faster than $X_{b}$. For this same reason, $\dot{m}_{v} / \dot{m}_{0}$ reduces with $\delta_{2}$ so that $\bar{h}_{m}$ de creases when $\delta_{2}$ increases, a result supported by Eqs. (39.b) and (40.b). These comments are in agreement with Eq. (43), for which an increase of $\delta_{2}$ decreases $\delta_{3}$ to such an extent that both the rate of absorption and the sheet thickness decreases.

The curves for $\delta_{2} \quad 10.2$ in Fig. 9 correspond to the reference case of a $\mathrm{LiBr} \mathrm{H}_{2} \mathrm{O}$ working pair. However, the curves associated to $\delta_{2} \quad 2.95$ have a resulting $\delta_{3} \quad 0.0216$ and, consequently, do not exactly match the results for the $\mathrm{LiNO}_{3} \mathrm{NH}_{3}$ solution $\left(\begin{array}{ll}\delta_{3} & 0.0201 \text { according to Table } 3\end{array}\right)$ depicted in diamond shaped points in Fig. 9.

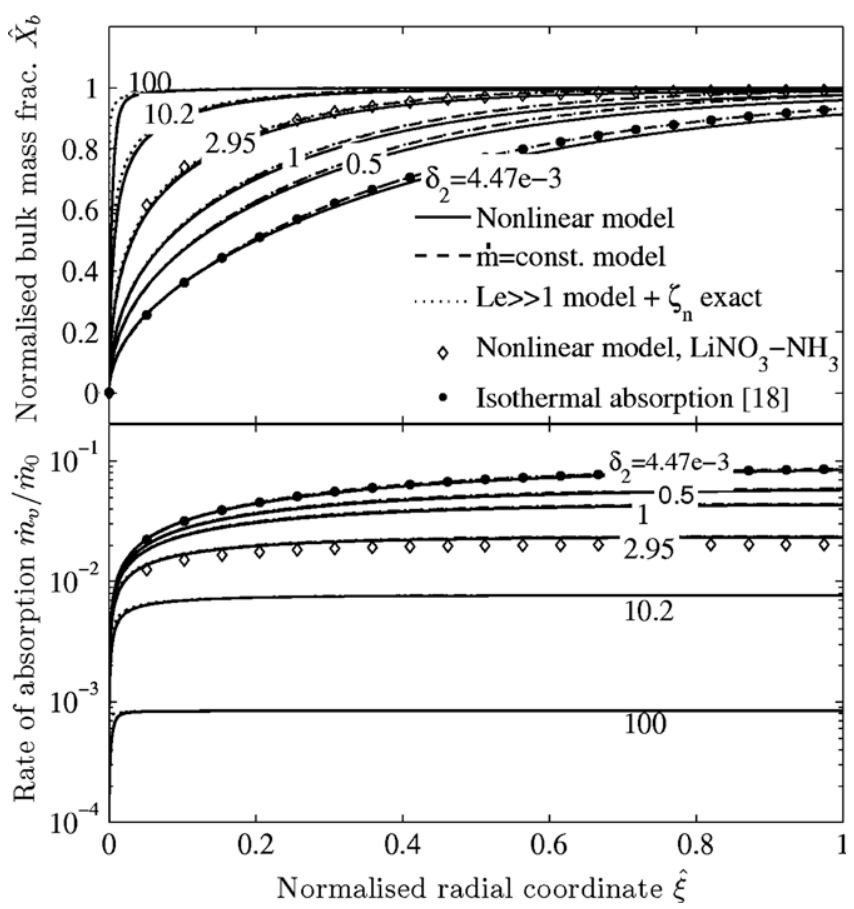

Fig. 9. Effect of the normalised heat of absorption $\delta_{2}$ on the bulk sheet mass fraction and on the rate of absorption for a solution with the same $L e$ and $c_{1} \Delta T_{\text {sub }} / X_{0}$ as the reference case of $\mathrm{LiBr}-\mathrm{H}_{2} \mathrm{O}$. Results for the isothermal absorption and for the working pair $\mathrm{LiNO}_{3}-\mathrm{NH}_{3}$ are also included.
With the aim to evaluate the models in close to isothermal con ditions, Fig. 9 also depicts the results for $\delta_{2} 4.47 \times 10^{3}$, corre sponding to the absorption of $\mathrm{CO}_{2}$ into an expanding water sheet. This particular value has been calculated using the equilibrium constant (deduced from Henry's law [45]) and the heat of absorp tion of $\mathrm{CO}_{2}$ into water at $25^{\circ} \mathrm{C}$ and at $1 \mathrm{~atm}$ [46]. In this special case, the heat of absorption is very small and the increase of tem perature in the solution is essentially negligible. This explains why the results for $\delta_{2} \quad 4.47 \times 10^{3}$ under the approximate models of $\dot{m}$ const. and $L e \gg 1$ agree perfectly with the isothermal absorp tion model presented by Tamir and Rachmilev [18].

It is relevant to notice that both the $\dot{m}$ const. model, the Le $\gg 1$ model and the Tamir and Rachmilev [18] model, are con structed under the hypothesis of the conservation of the flow rate in the sheet. This leads to a slight overprediction of $\hat{X}_{b}$ as compared to the nonlinear model at medium or large values of $\hat{\xi}$. This artificial overprediction of $\hat{X}_{b}$ occurs because these models provide a smaller mass in which to dissolve the absorbate than is provided in the nonlinear model. For analogous reasons, $\hat{T}_{b}$ will be also overpredicted in the $\dot{m}$ const. and in the $L e \gg 1$ models. Nevertheless, the overpredictions in $\hat{X}_{b}$ and $\hat{T}_{b}$ get reduced when $\delta_{2}$ increases toward values that are more commonly encountered in absorption refrigeration. This can be seen in Fig. 9 together with the inaccuracy in $\hat{X}_{b}$ that appears in the $L e \gg 1$ model at very small $\hat{\xi}$ and large $\delta_{2}$, as this model is not able to resolve large transversal gradients of temperature within the boundary layer region.

\subsection{Effect of initial subcooling}

The normalised subcooling $\delta_{3}$, Eq. (21), is the only parameter of the dimensionless equations that retains the effect of the solution's initial subcooling $\Delta T_{\text {sub }} . \delta_{3}$ has a weak effect on $\hat{X}$ and $\hat{T}$ since the normalisation of these variables partially hides the decrement in $X$ and $T$ caused by an increase of subcooling. Thus, Sh and $\overline{S h}$ are also weakly affected by $\delta_{3}$. Mathematically this is clear as $\delta_{3}$ is

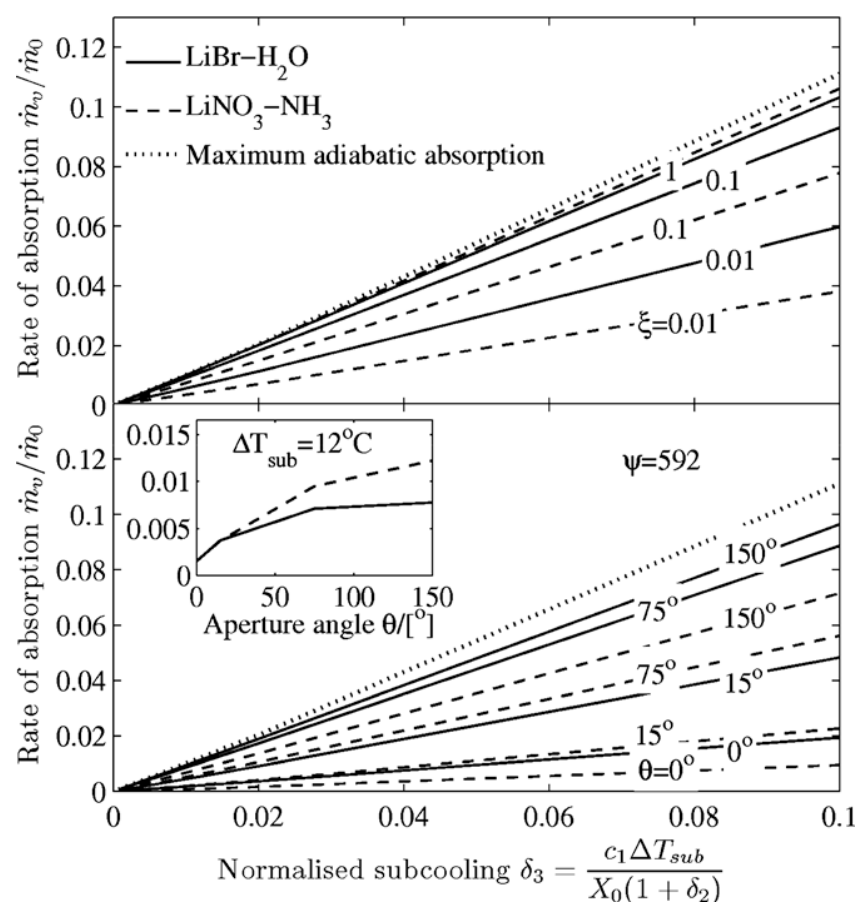

Fig. 10. Effect of subcooling on the rate of absorption into expanding sheets of different normalised radial lengths $\hat{\xi}$ and aperture angles $\theta$, for the pairs $\mathrm{LiBr}-\mathrm{H}_{2} \mathrm{O}$ and $\mathrm{LiNO}_{3}-\mathrm{NH}_{3}$. 
absent from the $\hat{X}$ and $\hat{T}$ equations for the $\dot{m}$ const. model and from the Eqs. (38). However, $\delta_{3}$ does possess a strong influence on derived variables such as $\hat{\varepsilon}$ and $\dot{m}_{v} / \dot{m}_{0}$.

Fig. 10 depicts the rate of absorption calculated with the nonlin ear model as a function of $\delta_{3}$ for selected values of $\hat{\xi}$. Results corre sponding to different aperture angles $\theta$ are also included in Fig. 10 for the initial cross section parameters of the reference case, $a_{0}$ and $\varepsilon_{0}$, and for a sheet length $y \quad 0.1 \mathrm{~m}(\psi \approx 592) . \hat{X}_{b}$ is constant in each curve of Fig. 10 and the absorption rate increases linearly with the initial subcooling. This fact fully agrees with the approximation provided by the right hand side of Eq. (43). The greater the $\hat{\xi}$ or $\theta$ selected for the curve, the higher the sensitivity of the absorption rate on the initial subcooling. Fig. 10 also contains the maximum rate of absorption for a pre set subcooling, which is reached when $\hat{\xi} \rightarrow \infty$ and $\hat{X}_{b} \rightarrow 1$ in, for example, infinitely long or extremely thin sheets. According to Eq. (43), the maximum rate of absorption that cannot be surpassed under adiabatic absorption is given by:

$\max \left(\frac{\dot{m}_{v}}{\dot{m}_{0}}\right) \quad \frac{\delta_{3}}{1 \quad \delta_{3}} \approx \frac{c_{1}}{X_{0}\left(1+\delta_{2}\right)} \Delta T_{\text {sub }}$

Eqs. (43) and (44) do not depend on the flow morphology and, conse quently, also apply to other adiabatic absorption configurations using sprays, films and bubbles. Under the reference conditions of Table 1 , Eq. (44) leads to $6.85 \times 10{ }^{4} \Delta T_{\text {sub }}$ for $\mathrm{LiBr} \mathrm{H}_{2} \mathrm{O}$ solutions and to $1.49 \times 10^{3} \Delta T_{\text {sub }}$ for $\mathrm{LiNO}_{3} \mathrm{NH}_{3}$. Therefore, taking the same $\Delta T_{\text {sub }}$ and a given $\hat{\xi}$ or $\theta$, the ammonia solution has a superior rate of absorp tion than the aqueous solution if the properties of Table 1 are taken for the comparison. This is also illustrated by the sub plot in Fig. 10 calculated for $12{ }^{\circ} \mathrm{C}$ of subcooling. When substituting for the subcool ing of the $\mathrm{LiBr} \mathrm{H}_{2} \mathrm{O}$ and $\mathrm{LiNO}_{3} \mathrm{NH}_{3}$ solutions (11.1 and $13.5^{\circ} \mathrm{C}$, respectively) the ammonia solution may potentially absorb up to 2.18 times the maximum vapour absorbed by the aqueous solution.

\section{Summary and conclusions}

In this study, the nonlinear system of equations resulting from modelling the coupled heat and mass transfer during non isother mal absorption of vapour into laminar expanding liquid sheets has been numerically integrated for two relevant examples of working pairs in absorption refrigeration: $\mathrm{LiBr} \mathrm{H}_{2} \mathrm{O}$ and $\mathrm{LiNO}_{3} \quad \mathrm{NH}_{3}$. Simpli fication of this nonlinear set of equations has been conducted by linearising them under the hypothesis of slowly increasing mass flow rate $(\dot{m} \approx$ const.), yielding results that compare well to the nonlinear model over the whole of the expanding sheet. In this re gard, the linear model has shown the equivalence between finite dilution effects and the introduction of an equivalent specific heat of absorption $L_{a b s}^{*}$. Further simplification of the linear equations for the frequent case of large Lewis number ( $L e \gg 1$ ) has been accom plished. The exceptionally simple analytical solution for the Le $\gg 1$ model has been facilitated by an explicit evaluation of the solution eigenvalues that has been proposed in this work. In the transition and in the fully developed region, the $L e \gg 1$ model with exact eigenvalues generates results of satisfactory quality for practical engineering applications, especially for $\mathrm{LiNO}_{3} \mathrm{NH}_{3}$ solutions. When approximate eigenvalues are used, the $L e \gg 1$ model is more suitable for $\mathrm{LiBr} \mathrm{H}_{2} \mathrm{O}$ solutions. In general, the $L e \gg 1$ model fails in describing the initial region of the sheet, but this is not the case for the boundary layer model adapted to this initial zone.

An analogy of the equations for expanding sheets and for non expanding sheets, or for falling films over adiabatic walls, has been used in this study to analytically show that expanding sheets always possess better performance than non expanding sheets. It is also shown that models relying on the mass flow rate conservation assumption slightly overpredict the absorbed mass for working pairs with small normalised heat of absorption $\delta_{2}$. Additionally, the local and mean Sherwood numbers have been characterised and their closed form analytical expressions determined for the boundary layer and fully developed regions. In particular, it is shown that the mean Sherwood number in expanding sheets is $4 / 3\left(\delta_{2} L e^{1 / 2}+1\right)$ times the local Sherwood number in the fully developed region. Both dimensionless groups only depend on the initial absorbent mass fraction and the normalised heat of absorp tion. For the same initial subcooling, and under the conditions of adi abatic absorption selected in this research, the maximum rate of absorption for $\mathrm{LiNO}_{3} \quad \mathrm{NH}_{3}$ solutions is larger than for $\mathrm{LiBr} \mathrm{H}_{2} \mathrm{O}$. All these parametric expressions may be applied to the design of adia batic absorbers, and other processes such as desorption and direct condensation on sheets may benefit from the models and proce dures presented in this study.

\section{Acknowledgements}

The authors wish to express their gratitude to Dr. M. Venegas for her useful comments. This work has been partially funded by the Spanish Government Research Grants DPI 200202439 and ENE 200508255 CO2 02, as well as by the Autonomous Commu nity of Madrid \& UC3M through CCG07 UC3M/AMB 3412 project. Their contribution is greatly appreciated.

\section{Appendix A. Mass fraction and temperature in complete adiabatic saturation}

Absorption under adiabatic conditions decreases the absorbent bulk mass fraction $X_{b}$ and increases the bulk solution temperature $T_{b}$ toward limiting values. These limiting values are the minimum absorbent mass fraction $X_{\infty}$ and the maximum temperature $T_{\infty}$, which cannot be surpassed by the bulk variables unless, con tradicting the adiabatic condition, the solution is cooled during the absorption process. It is possible to estimate $X_{\infty}$ and $T_{\infty}$ by means of the following global mass and energy balances performed for an ideal adiabatic absorber in which the solution reaches com plete saturation:

$\dot{m}_{0}+\dot{m}_{v \infty} \quad \dot{m}_{\infty}$

$\dot{m}_{0} h_{0}+\dot{m}_{v \infty} h_{v} \quad \dot{m}_{\infty} h_{\infty}$

where $\dot{m}_{0}, \dot{m}_{\infty}$ are the liquid solution mass flow rate at the inlet and outlet of the ideal adiabatic absorber (respectively) and $\dot{m}_{v \infty}$ is the mass flow rate of the absorbed vapour. In Eq. (A.2) $h_{v}, h_{0}$ and $h_{\infty}$ stand for the enthalpies of the absorbent vapour, the solution at the chamber inlet, and the solution at the outlet, respectively. Note that the specific heat of absorption can be approximated by

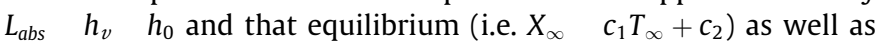
homogeneity take place at the outlet. Owing to the species mass fraction homogeneity at the chamber inlet and outlet, the mass flow rates are intrinsically related to the absorbent mass fraction through $\dot{m}_{0} / \dot{m}_{\infty} \quad X_{\infty} / X_{0}$. From Eqs. (A.1) and (A.2), it is straightfor ward to deduce next the approximate expressions for the absorbent mass fraction and solution temperature reached under complete saturation in adiabatic absorption:

$$
\begin{array}{ll}
X_{\infty} & \frac{X_{0} \cdot\left(\delta_{1}+X_{e q}\left(T_{0}\right)\right)}{\delta_{1}+X_{0}} \\
T_{\infty} & \frac{X_{\infty} c_{2}}{c_{1}}
\end{array}
$$

where $X_{e q}\left(T_{0}\right)$ is the equilibrium absorbent mass fraction, Eq. (10), at the solution inlet temperature $T_{0}$, and where $\delta_{1}$ is the dimension less heat of absorption described in Eq. (19). Any point in a solution sheet of infinite length would theoretically reach $X_{\infty}$ and $T_{\infty}$ be cause in this configuration the exposure time of the solution to 
the vapour is limitless and diffusion eliminates transversal gradi ents. Then, $\hat{X} \rightarrow 1$ and $\hat{T} \rightarrow 1$ if $\hat{\xi} \rightarrow \infty$. According to Eqs. (A.3) and (A.4), and for the properties presented in Table 1 , the maximum increments of absorbate bulk mass fraction, i.e. $X_{0} \quad X_{\infty}$, and bulk temperature, $T_{\infty} \quad T_{0}$, are, respectively, restricted to $4.82 \times 10^{3}$ and $10.1{ }^{\circ} \mathrm{C}$ for $\mathrm{LiBr} \mathrm{H}_{2} \mathrm{O}$ solutions, and to $1.27 \times 10^{2}$ and $10.08{ }^{\circ} \mathrm{C}$ for $\mathrm{LiNO}_{3} \mathrm{NH}_{3}$ solutions.

\section{References}

[1] K.E. Herold, R. Radermacher, S. Klein, Absorption Chillers and Heat Pumps, CRC Press, New York, 1996.

[2] F. Ziegler, Recent developments and future prospects of sorption heat pumps systems, Int. J. Therm. Sci. 38 (3) (1999) 191-208.

[3] G. Grossman, Adiabatic absorption and desorption for improvement of temperature-boosting absorption heat pumps, ASHRAE Trans. 88 (2) (1982) 359-367.

[4] S.G. Simpson, S. Lynn, Vacuum-spray stripping of sparingly soluble gases from aqueous solutions: Part I. Mass transfer from streams issuing from hydraulic nozzles, AIChE J. 23 (5) (1977) 666-673.

[5] N. Dombrowski, R.P. Fraser, A photographic investigation into the disintegration of liquid sheets, Philos. Trans. R. Soc. Lond. A 247 (924) (1954) 101-130.

[6] N. Dombrowski, D. Hasson, D.E. Ward, Some aspects of liquid flow through fan spray nozzles, Chem. Eng. 12 (1) (1960) 35-50.

[7] G.I. Taylor, The dynamics of thin sheets of fluid II. Water in fluid sheets, Proc. $R$. Soc. Lond. A 253 (1274) (1959) 296-312.

[8] G.I. Taylor, The dynamics of thin sheets of fluid III. Disintegration of fluid sheets, Proc. R. Soc. Lond. A 253 (1274) (1959) 313-321.

[9] G.I. Taylor, Formation of thin flat sheets of water, Proc. R. Soc. A 259 (1296) (1960) 1-17.

[10] S. Weinberg, Heat transfer to low pressure sprays of water steam atmosphere, Proc. Inst. Mech. Eng. 1B (6) (1952) 240-253.

[11] S.Y. Lee, R.S. Tankin, Study of liquid spray (water) in a condensable environment (steam), Int. J. Heat Mass Transfer 27 (3) (1984) 363-374.

[12] M. Takahashi, A.K. Nayak, S. Kitagawa, H. Murakoso, Heat transfer in direct contact condensation of steam to subcooled water spray, J. Heat Transfer 123 (4) (2001) 703-710.

[13] F. Mayinger, A. Chávez, Measurement of direct-contact condensation of pure saturated vapour on an injection spray by applying pulsed laser holography, Int. J. Heat Mass Transfer 35 (3) (1992) 691-702.

[14] D. Hasson, D. Luss, U. Navon, An experimental study of steam condensation on laminar water sheet, Int. J. Heat Mass Transfer 7 (9) (1964) 983-1001.

[15] A. Tamir, D. Hasson, Evaporation and condensation coefficient of water, Chem. Eng. J. 2 (3) (1971) 200-211.

[16] D. Hasson, D. Luss, R. Peck, Theoretical analyses of vapour condensation on laminar liquid jets, Int. J. Heat Mass Transfer 7 (9) (1964) 969-981.

[17] S.Y. Lee, R.S. Tankin, Study of liquid spray (water) in a non-condensable environment (air), Int. J. Heat Mass Transfer 27 (3) (1984) 363-374.

[18] A. Tamir, I. Rachmilev, Absorption of carbon dioxide in a rapid laminar fanshaped water sheet, Chem. Eng. J. 4 (1) (1972) 87-90.

[19] S.G. Simpson, S. Lynn, Vacuum-spray stripping of sparingly soluble gases from aqueous solutions: Part II. Stripper design using jet impingement nozzles, AIChE J. 23 (5) (1977) 673-679.

[20] W. Ryan, F. Ruiz, J. Wurm, Model development and verification of spray absorption for gas driven cooling systems, in: Proceedings of the International Gas Research Conference, Cannes, France, 1995, pp. 1483-1493.

[21] G. Grossman, Heat and mass transfer in film absorption, in: N.P. Cheremisinoff (Ed.), Handbook of Heat and Mass Transfer, vol. 2, Gulf Publishing, 1986, pp. 211-257 (Chapter 6).

[22] J.D. Killion, S. Garimella, A critical review of models of coupled heat and mass transfer in falling film absorption, Int. J. Refrigerat. 24 (8) (2001) 755797.
[23] M. Venegas, M. Izquierdo, P. Rodríguez, A. Lecuona, Heat and mass transfer during absorption of ammonia vapour by $\mathrm{LiNO}_{3}-\mathrm{NH}_{3}$ solution droplets, Int. J. Heat Mass Transfer 47 (12-13) (2004) 2653-2667.

[24] R.M. DiGuilio, R.J. Lee, S.M. Jeter, A.S. Teja, Properties of lithium bromidewater solutions at high temperatures and concentrations - I. Thermal conductivity, ASHRAE Trans. 96 (1) (1990) 702-708.

[25] L.A. McNeely, Thermodynamic properties of aqueous solutions of lithium bromide, ASHRAE Trans. 85 (1) (1979) 413-434.

[26] ASHRAE, Fundamentals Handbook, ASHRAE, Atlanta, 2005.

[27] I. Morioka, M. Kiyota, A. Ousaka, T. Kobayashi, Analysis of steam absorption by subcooled droplet of aqueous solution of LiBr, JSME Int. J. Ser. II 35 (3) (1992) 458-464.

[28] J. Fernández-Seara, F.J. Uhía, J. Sieres, Analysis of an air cooled ammonia-water vertical tubular absorber, Int. J. Therm. Sci. 46 (1) (2007) 93-103.

[29] R.J. Demyanovich, J.R. Bourne, Rapid micromixing by the impingement of thin liquid sheets 1 . A photographic study of the flow pattern, Ind. Eng. Chem. Res. 28 (6) (1989) 825-830.

[30] C.J. Clark, N. Dombrowski, The dynamics of the rim of a fan spray sheet, Chem. Eng. Sci. 26 (11) (1971) 1949-1952.

[31] R.B. Bird, W.E. Stewart, E.N. Lightfoot, Transport Phenomena, second ed., John Wiley, New York, 2001.

[32] N. Brauner, Non-isothermal vapour absorption into falling film, Int. J. Heat Mass Transfer 34 (3) (1991) 767-784.

[33] G. Grossman, Simultaneous heat and mass transfer in film absorption under laminar flow, Int. J. Heat Mass Transfer 26 (3) (1983) 357-371.

[34] A. Acosta, N. García, P. Rodríguez, Coupled heat and mass transfer during absorption of water vapour into $\mathrm{LiBr}-\mathrm{H}_{2} \mathrm{O}$ solution fan sheets, in: Proceedings of CHT-08 ICHMT International Symposium on Advances in Computational Heat Transfer, May 11-16, Marrakesh, Morocco, 2008, CHT-08-269 pp.

[35] V.E. Nakoryakov, N.S. Bufetov, N.I. Grigorieva, R.A. Dekhtyar, I.V. Marchuk, Vapor absorption by immobile solution layer, Int. J. Heat Mass Transfer 47 (6) (2004) 1525-1533.

[36] B.J.C. van der Wekken, R.H. Wassenaar, Simultaneous heat and mass transfer accompanying absorption in laminar flow over a cooled wall, Int. J. Refrigerat. 11 (2) (1988) 70-77.

[37] V.E. Nakoryakov, N.I. Grigorieva, Calculation of heat and mass transfer in nonisothermal absorption on the initial portion of a downflowing film, Theor. Found. Chem. Eng. 14 (4) (1980) 483-488.

[38] D.A. Anderson, J.C. Tannehill, R.H. Pletcher, Computational Fluid Mechanics and Heat Transfer, Taylor \& Francis, 1984.

[39] V.E. Nakoryakov, N.I. Grigorieva, Exact solution to the problem of combined heat and mass transfer at film absorption, J. Eng. Phys. 33 (5) (1977) 13491353.

[40] V.E. Nakoryakov, N.I. Grigorieva, L.V. Potaturkina, Analysis of exact solutions to heat- and mass-transfer problems for absorption with films or streams, Theor. Found. Chem. Eng. 31 (2) (1997) 119-126.

[41] R. Clift, J.R. Grace, M.E. Weber, Bubbles, Drops and Particles, Academic Press, New York, 1978

[42] D. Arzoz, P. Rodríguez, M. Izquierdo, Experimental study on the adiabatic absorption of water vapor into $\mathrm{LiBr}-\mathrm{H}_{2} \mathrm{O}$ solutions, Appl. Therm. Eng. 25 (5-6) (2005) 797-811.

[43] H. Le Goff, A. Ramadane, M. Barkaoui, Y. Chen, P. Le Goff, Modeling the coupled heat and mass transfer in a falling film: application to absorbers and desorbers in absorption heat pumps, in: Heat Transfer, Proceedings of the International Heat Transfer Conference, vol. 4, Hemisphere Publications, New York, 1986, pp. 1971-1976.

[44] T.K. Sherwood, R.L. Pigford, C.R. Wilke, Mass Transfer, McGraw-Hill, New York, 1975.

[45] D.B. Spalding, Convective Mass Transfer; an Introduction, Edward Arnold, London, 1963.

[46] R.L. Berg, C.E. Vanderzee, Thermodynamics of carbon dioxide and carbonic acid: (a) the standard enthalpies of solution of $\mathrm{Na}_{2} \mathrm{CO}_{3}(\mathrm{~s}), \mathrm{NaHCO}_{3}(\mathrm{~s})$, and $\mathrm{CO}_{2}(\mathrm{~g})$ in water at $298.15 \mathrm{~K}$; (b) the standard enthalpies of formation, standard Gibbs energies of formation, and standard entropies of $\mathrm{CO}_{2}(\mathrm{aq}), \mathrm{HCO}_{3}$ (aq), $\mathrm{CO}_{3}{ }^{2}$ (aq), $\mathrm{NaHCO}_{3}(\mathrm{~s}), \mathrm{Na}_{2} \mathrm{CO}_{3}(\mathrm{~s}), \mathrm{Na}_{2} \mathrm{CO}_{3} \cdot \mathrm{H}_{2} \mathrm{O}(\mathrm{s})$, and $\mathrm{Na}_{2} \mathrm{CO}_{3} \cdot 10 \mathrm{H}_{2} \mathrm{O}(\mathrm{s})$, J. Chem. Thermodyn. 10 (12) (1978) 1113-1136. 